\title{
Catalytic robustness and torque generation of the $F_{1}$-ATPase
}

\author{
Hiroyuki Noji $^{1}$ - Hiroshi Ueno ${ }^{1}$ Duncan G. G. McMillan ${ }^{1}$
}

Received: 30 January 2017 / Accepted: 13 March 2017 / Published online: 25 March 2017

(C) The Author(s) 2017. This article is published with open access at Springerlink.com

\begin{abstract}
The $\mathrm{F}_{1}$-ATPase is the catalytic portion of the $\mathrm{F}_{\mathrm{o}} \mathrm{F}_{1}$ ATP synthase and acts as a rotary molecular motor when it hydrolyzes ATP. Two decades have passed since the singlemolecule rotation assay of $\mathrm{F}_{1}$-ATPase was established. Although several fundamental issues remain elusive, basic properties of F-type ATPases as motor proteins have been well characterized, and a large part of the reaction scheme has been revealed by the combination of extensive structural, biochemical, biophysical, and theoretical studies. This review is intended to provide a concise summary of the fundamental features of $F_{1}$-ATPases, by use of the well-described model $\mathrm{F}_{1}$ from the thermophilic Bacillus PS3 $\left(\mathrm{TF}_{1}\right)$. In the last part of this review, we focus on the robustness of the rotary catalysis of $\mathrm{F}_{1}$-ATPase to provide a perspective on the re-designing of novel molecular machines.
\end{abstract}

Keywords $\mathrm{F}_{1}$-ATPase $\cdot$ ATP synthase $\cdot$ Single-molecule techniques $\cdot$ Molecular motor

\section{Introduction}

The $\mathrm{F}_{\mathrm{o}} \mathrm{F}_{1}$ ATP synthase is a ubiquitous enzyme, spreading across all kingdoms of biology. It is found in the inner membrane of mitochondria, the thylakoid membrane of chloroplasts, and the plasma membranes of bacteria and some archaea. However, most archaea, and some eukaryotic bacteria,

Hiroyuki Noji

hnoji@appchem.t.u-tokyo.ac.jp

1 Department of Applied Chemistry, Graduate School of Engineering, The University of Tokyo, Tokyo 113-8656, Japan employ the evolutionary related V-type ATPase as an ATP synthase (Yoshida et al. 2001). $\mathrm{F}_{\mathrm{o}} \mathrm{F}_{1}$ ATP synthases catalyze ATP synthesis using ADP and inorganic phosphate as substrates. ATP synthesis is a thermodynamically uphill reaction. To catalyze ATP synthesis, the $\mathrm{F}_{\mathrm{o}} \mathrm{F}_{1}$ ATP synthase utilizes an electrochemical potential of protons or sodium ions across biological membranes (proton motive force, pmf, or sodium motive force, $s m f$ ) as the driving force (Abrahams et al. 1994; Dimroth et al. 2006; Yoshida et al. 2001). The use of chemiosmotic catalysis by the ATP synthase is such a prevalent reaction in biology that the $\mathrm{F}_{\mathrm{o}} \mathrm{F}_{1}$ is widely considered to be one of the most ancient enzymes. A scenario on the last universal common ancestor (LUCA) proposes that an ancestor molecular complex of ATP synthase prebiotically emerged to confer energy production for LUCA (Lane and Martin 2012), although there are arguments against this scenario (Jackson 2016).

$F_{o}$ and $F_{1}$

The $\mathrm{F}_{\mathrm{o}} \mathrm{F}_{1}$ ATP synthase is a multisubunit membraneintegrated enzyme with a molecular weight of $>500 \mathrm{kDa}$. This enzyme is composed of two structurally and functionally distinct portions, $\mathrm{F}_{\mathrm{o}}$ and $\mathrm{F}_{1}$, each of which can be considered to be a rotary molecular motor (Junge et al. 1997; Noji and Yoshida 2001; Oster and Wang 2000). The $\mathrm{F}_{\mathrm{o}}(\sim 120 \mathrm{kD})$ is the membrane-embedded portion of ATP synthase (Fig. 1). Bacterial $\mathrm{F}_{\mathrm{o}}$ have the simplest subunit composition, $a_{1} b_{2} c_{\mathrm{x}}$, where the number of $c$ subunits varies from 8 to 15 among different organisms, yet remains invariant within individual organisms (Ballhausen et al. 2009; Matthies et al. 2009; Meier et al. 2005b; Pogoryelov et al. 2007; Stock et al. 1999). $F_{o}$ mediates proton or sodium ion translocation across the membrane and, in doing so, the $c$ oligomer ring rotates against the $a b_{2}$ stator complex. The $a$ subunit is comprised of 


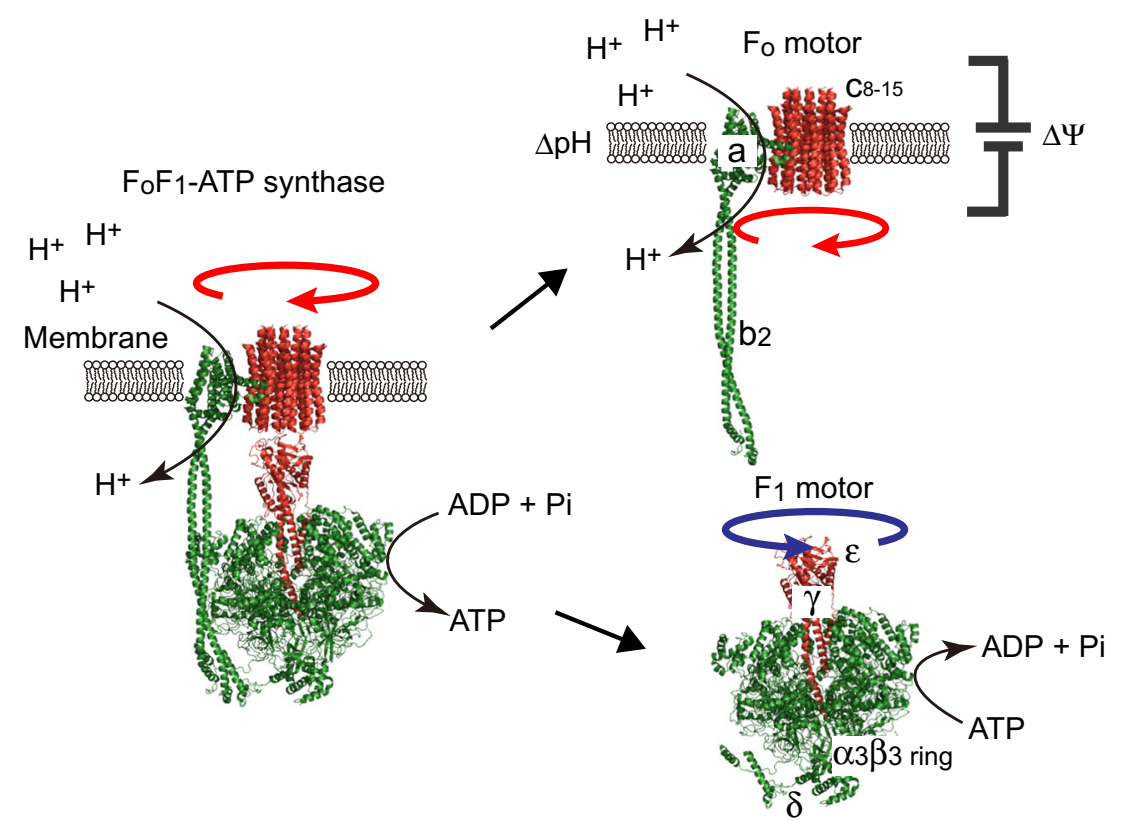

Fig. 1 Two rotary motors of $\mathrm{F}_{\mathrm{o}} \mathrm{F}_{1}$-ATP synthase. Schematic models of the $\mathrm{F}_{\mathrm{o}} \mathrm{F}_{1}$-ATP synthase. The structural models of $\mathrm{F}_{\mathrm{o}} \mathrm{F}_{1}$ (PDB ID: 5T4O) (Sobti et al. 2016) are shown as cartoon representation. The rotor and stator parts are shown in red and green, respectively. The $\mathrm{F}_{\mathrm{o}} \mathrm{F}_{1}$-ATP synthase is composed of two tethered rotary motors, $F_{o}$ and $F_{1}$, each driven by two different fuels. The subunit composition of bacterial $F_{1}$ and $\mathrm{F}_{\mathrm{o}}$ are $\alpha_{3} \beta_{3} \gamma \delta \varepsilon$ and $a b_{2} c_{\mathrm{x}}$ (where $\mathrm{x}$ is the copy number of $c$ subunits, which varies from 8 to 15 in different species), respectively. The membrane-embedded $\mathrm{F}_{\mathrm{o}}$ motor rotates the $c$-ring (rotor) against the

two half-channels, one exposed to each side of the membrane. Protons/sodium ions enter through one half-channel (entry channel) and are delivered to the $c$ oligomer. Upon one $360^{\circ}$ rotation of the $c$ oligomer, each proton/sodium ion is redelivered to the $a$ subunits through the other half-channel (exit channel) and released into the cell (Allegretti et al. 2015; Junge et al. 1997). Due to the difficulties with purification, handling, and analysis of the membrane-embedded $F_{o}$, the functional and structural analyses of the $\mathrm{F}_{\mathrm{o}}$ has long lagged behind the understanding we have of the $F_{1}$. Recently, the structural information of how the $a$ subunit forms the proton translocation path together with the $c$ subunit has been available (Allegretti et al. 2015). However, together with crystallographic analysis, the recent advances in single-particle analysis together with electron microscopy are rapidly advancing our understanding of the structural detail of $\mathrm{F}_{\mathrm{o}}$ (Morales-Rios et al. 2015). However, this review is not focused on the latest functional and structural studies on $\mathrm{F}_{\mathrm{o}}$. Readers are encouraged to visit other reviews that more than adequately address this (Junge and Nelson 2005; Kühlbrandt and Davies 2016).

\section{F $_{1}$-ATPase}

The $F_{1}$ is the water-soluble and catalytic portion of the $F_{0} F_{1}$ ATP synthase. The subunit composition of bacterial $F_{1}$ is $a b_{2}$ (stator), clockwise when viewed from the membrane side, which is driven by $p m f$ consisting of membrane potential $(\Delta \Psi)$ and proton concentration gradient $(\Delta \mathrm{pH})$. The $\mathrm{F}_{1}$ is an ATP-driven rotary motor in which the $\gamma$ subunit (rotor) rotates against the $\alpha_{3} \beta_{3}$-ring (stator). The $\varepsilon$ subunit binds to the protruding part of the $\gamma$ subunit. The $\delta$ binds to the bottom of the $\alpha_{3} \beta_{3}$-ring. Note that the rotational direction of $F_{1}$ is opposite to that of $\mathrm{F}_{\mathrm{o}}$. In the whole complex of $\mathrm{F}_{\mathrm{o}} \mathrm{F}_{1}, \mathrm{~F}_{\mathrm{o}}$ reverses the rotation of $\mathrm{F}_{1}$, leading to ATP synthesis from $\mathrm{ADP}$ and $\mathrm{Pi}$

$\alpha_{3} \beta_{3} \gamma_{1} \delta_{1} \varepsilon_{1}$. The $F_{1}$ domain of most described enzymes show high ATP hydrolytic activity, typically in the range of $10^{2}-10^{3}$ turnover/s (Bilyard et al. 2013; McMillan et al. 2016; Spetzler et al. 2006; Yasuda et al. 2001). For this reason, the $F_{1}$ is frequently referred to as the ' $\mathrm{F}_{1}$-ATPase'. However, the ATP hydrolytic activities of some the $\mathrm{F}_{1}$ are latent and inhibited in various ways (Cingolani and Duncan 2011; Keis et al. 2006; McMillan et al. 2007; Morales-Rios et al. 2015). This suppressive regulation is thought to avoid the futile consumption of ATP in a cell (Feniouk and Yoshida 2008). The minimum complex of $F_{1}$ as a rotary molecular motor is the $\alpha_{3} \beta_{3} \gamma_{1}$ subcomplex, in which the $\gamma$ subunit is inserted in the central cavity of the $\alpha_{3} \beta_{3}$-ring. Crystal structures of $F_{1}$ have revealed fundamental aspects of $F_{1}$ (Fig. 2; Abrahams et al. 1994). The catalytic reaction centers of $F_{1}$ for ATP hydrolysis/synthesis are located at the $\alpha \beta$ interfaces (Weber and Senior 1997). Most of the amino acid residues that form the ATP-binding pocket reside in the $\beta$ subunit, while the $\alpha$ subunit possesses one catalytically critical arginine residue (Ahmad and Senior 2005; Hayashi et al. 2012; Kagawa et al. 2004; Komoriya et al. 2012).

Upon ATP hydrolysis, the $\beta$ subunit undergoes a large conformational change to induce the unidirectional rotation of the $\gamma$ subunit, counterclockwise when viewed from the membrane side (Abrahams et al. 1994; Masaike et al. 2008; Noji et al. 1997). The $\varepsilon$ subunit binds to the protruding domain of the $\gamma$ to 

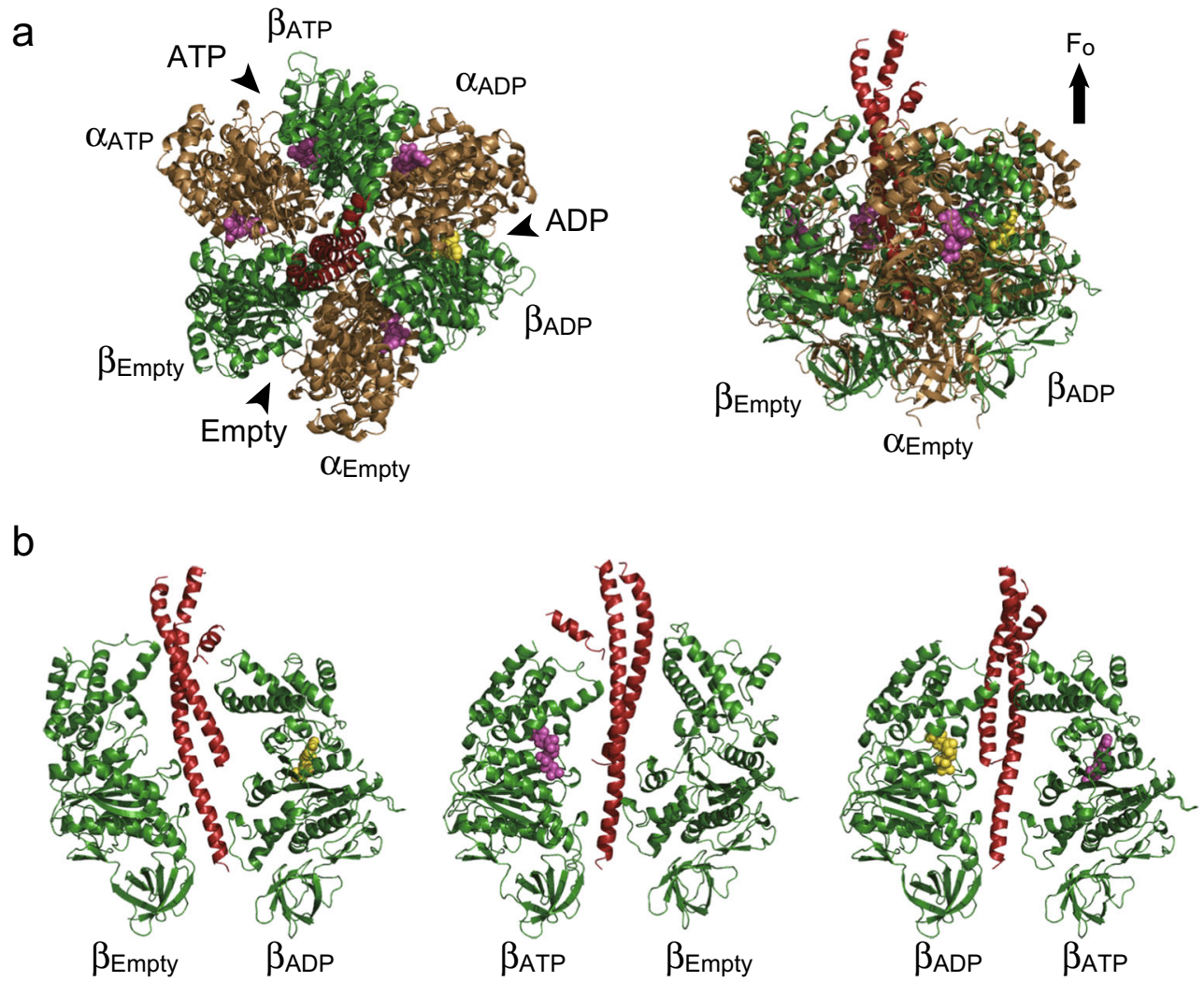

Fig. 2 Crystal structures of $F_{1}$. The crystal structures of $F_{1}$ from bovine mitochondria (PDB ID: 1BMF) (Abrahams et al. 1994) are shown in cartoon diagrams as a top view from the membrane side (a, left) and as a side view (a, right and $\mathbf{b})$. a The $\alpha, \beta$, and $\gamma$ subunits are shown in dark yellow, green, and red, respectively. The bound AMP-PNP and ADP are shown in magenta and yellow, respectively. The catalytic sites are located at the interfaces between $\alpha$ and $\beta$ subunits (black arrowheads), which are mainly harbored by the $\beta$ subunits. Each carries AMP-PNP, ADP, or none. Therefore, each $\beta$ subunit catalytic site at any one point in time is designated as $\beta_{\mathrm{ATP}} \beta_{\mathrm{ADP}}$, or $\beta_{\mathrm{Empty}}$, respectively. The non-catalytic sites

are located at the other interfaces, all of which are occupied with AMPPNP. Each $\alpha$ subunit forming a catalytic site is designated as $\alpha_{\mathrm{ATP}}, \alpha_{\mathrm{ADP}}$, or $\alpha_{\text {Empty }}$, respectively. The protruding part of the $\gamma$ subunit is directed toward the $F_{o}$ side. $\mathbf{b}$ Three $\beta-\beta$ pairs with different nucleotide states are shown with the $\gamma$ subunit. Both $\alpha$ and $\beta$ subunits are composed of the $\mathrm{N}$ terminal domain, nucleotide-binding domain, and $\mathrm{C}$-terminal domain (from bottom to top). $\beta_{\text {Empty }}$ takes an open conformation, and both $\beta_{\text {ATP }}$ and $\beta_{\text {ADP }}$ take a closed conformation with bound nucleotide. The C-terminal domain of the closed $\beta$ subunit appears to push the $\gamma$ subunit

form the binding interface to $\mathrm{F}_{\mathrm{o}}$. The $\varepsilon$ subunit is also known to be a catalytic regulator (Cingolani and Duncan 2011; Feniouk et al. 2010; Hara et al. 2001; Keis et al. 2006; Tsunoda et al. 2001). The $\delta$ subunit attaches to the bottom tip of the $\alpha_{3} \beta_{3}$ ring (Dunn and Chandler 1998; Ogilvie et al. 1997).

\section{Coupling between $F_{1}$ and $F_{o}$}

In the complete complex, $F_{o}$ and $F_{1}$ are connected via two stalks: the central and the peripheral. The central stalk is the rotary shaft of $\mathrm{F}_{\mathrm{o}} \mathrm{F}_{1}$ ATP synthase, composed of the rotor parts of $F_{o}$ and $F_{1}$ (Stock et al. 1999; Wächter et al. 2011). Together, the $\gamma \varepsilon$ complex and the $c$-ring form the combined entity of the rotary shaft. $\mathrm{F}_{\mathrm{o}}$ and $\mathrm{F}_{1}$ form the second stalk, termed the peripheral stalk. This is comprised of the $\delta$ subunit and the $b_{2}$ dimer. The peripheral stalk is located on the lateral side of ATP synthase and firmly holds both of the stator parts of $F_{1}$ and $F_{o}$ from the outside, spanning from the bottom tip of $F_{1}$ to the opposite side of the lipid bilayer (Rodgers and Capaldi 1998;
Stock et al. 1999). In the ATP synthase, $F_{o}$ and $F_{1}$ exert rotational torque against each other (Junge et al. 1997; Noji and Yoshida 2001; Oster and Wang 2000). Under physiological conditions where $p m f$ is sufficient and the rotational torque of $F_{o}$ surpasses that of $F_{1}, F_{o}$ rotates the $\gamma$ subunit in the reverse direction, a clockwise rotation viewed from the membrane side (from the top of Fig. 1).

The reverse rotation of $F_{1}$ induces the reverse conformational change of the $\alpha_{3} \beta_{3}$-ring, leading to the reverse reaction of ATP hydrolysis (ATP synthesis). When the free energy of ATP hydrolysis dominates, and the $\mathrm{F}_{1}$ generates a larger torque than $\mathrm{F}_{\mathrm{o}}, \mathrm{F}_{1}$ reverses the rotation of $\mathrm{F}_{\mathrm{o}}$ to induce active proton pumping and build up $p m f$ across the membrane. Thus, $\mathrm{F}_{\mathrm{o}}$ and $\mathrm{F}_{1}$ interchange the energy between $p m f$ and free energy of ATP hydrolysis via the mechanical rotation of the rotor complex. For efficient energy interconversion, the central stalk and the peripheral stalk have to be resistant against mechanical torsional stress (Wächter et al. 2011). The $\delta b_{2}$ subcomplex is known to form a stable complex (McLachlin 
et al. 1998). Regarding the central stalk, although the $c$ subunit oligomer ring has multiple symmetric binding sites for the $\gamma \varepsilon$ complex (Müller et al. 2004; Schulenberg et al. 1999; Watts et al. 1995), there have been, to date, no reports of slippage at the interface of the $\gamma \varepsilon$ subcomplex and the $c$-ring. On the other hand, some studies suggest that the stalks are twisted in the ATP synthase at work. Although the elasticity of the peripheral stalk has been reported previously (Junge et al. 2009), recent studies suggest that the $\gamma$ subunit of the central stalk is twisted (Okazaki and Hummer 2015; Vahidi et al. 2016). It remains elusive how the elasticity of the stalks contribute to energy transduction within the ATP synthase.

\section{$\mathbf{H}^{+} /$ATP stoichiometry}

Since the stoichiometry of protons and ATP per rotation is not the same, the direction of the reaction, i.e., rotation is determined by the balance of total $\Delta G_{\mathrm{ATP}}$ and pmf coupled with one turn of the $\gamma \varepsilon-c$-ring spindle:

$m \times \Delta G_{A T P}$ and $n \times p m f$

where $m$ and $n$ represent the number of ATP and protons coupled with a single turn.

All of $F_{1}$, of which crystal structures are available, show a pseudo three-fold symmetry in the $\alpha \beta$ stator ring (Abrahams et al. 1994; Böttcher and Gräber 2000; Cingolani and Duncan 2011; Stocker et al. 2005). Thus, the stoichiometry of $F_{1}(m)$ should be 3 ATPs per turn. Actually, all of the $\mathrm{F}_{1}$ tested in the rotation assay show stepping rotation with $120^{\circ}$ intervals (Bilyard et al. 2013; Konno et al. 2006; McMillan et al. 2016; Suzuki et al. 2014; Yasuda et al. 1998). Thus, it is well established that $m=3$.

On the other hand, the stoichiometry of proton translocation per turn $(n)$ is variable between species, yet is invariant within species. It is assumed that $n$ is determined by the number of $c$ subunits in the oligomeric $c$-ring rotor of $F_{o}$ (Junge et al. 1997). While the bacterial $F_{o}$ has generally $10-15 c$ subunits in the oligomer ring (Ballhausen et al. 2009; Matthies et al. 2009; Meier et al. 2005a; Pogoryelov et al. 2005, 2009), mammalian mitochondrial $\mathrm{F}_{\mathrm{o}}$ has 8 (Watt et al. 2010) and, depending on the origin, chloroplasts have 13-15 subunits in the $c$-ring (Pogoryelov et al. 2007). Several groups reported that $F_{0} F_{1}$ from Escherichia coli makes the rotation with 10 steps, which is consistent with the number of $c$ subunits being 10 (Ballhausen et al. 2009; Düser et al. 2009; Ishmukhametov et al. 2010). It should be noted that the apparent number of rotation steps of $\mathrm{F}_{\mathrm{o}} \mathrm{F}_{1}$ depends on the experimental conditions, because 10-step rotation should be observed only when the proton translocation or conformational change of $F_{o}$ determines the overall rate of rotation. Otherwise, step rotation of $\mathrm{F}_{\mathrm{o}} \mathrm{F}_{1}$ should represent the structural symmetry of $\mathrm{F}_{1}$, as previously reported (Watanabe et al. 2013).
Thus, the structural symmetry of the $c$-ring and $\alpha_{3} \beta_{3}$-ring would set the stoichiometry ratio of protons against ATP as:

$H^{+} / A T P=n / m=n / 3$.

When we consider possible uncoupling rotation of $\mathrm{F}_{\mathrm{o}}$, i.e., slipped rotation without accompanying proton translocation, this value sets the upper limit of the $\mathrm{H}^{+} / \mathrm{ATP}$ ratio. Before structural information on the $c$-ring became available, the $\mathrm{H}^{+}$/ATP ratio was considered to be an integer, such as 3 or 4 , and to be common among species. Recent studies show that this is not the case. The $\mathrm{H}^{+} / \mathrm{ATP}$ ratio estimated from the structure is 2.7 for mammalian mitochondrial $F_{1}, 3.3$ for E. coli $\mathrm{F}_{\mathrm{o}} \mathrm{F}_{1}$, and 4 or more for chloroplast $\mathrm{F}_{\mathrm{o}} \mathrm{F}_{1}$ (Ballhausen et al. 2009; Pogoryelov et al. 2005; Watt et al. 2010). Biochemical analysis supports that $\mathrm{F}_{\mathrm{o}} \mathrm{F}_{1}$ with a larger number of the $c$ subunit shows a larger $\mathrm{H}^{+}$/ATP ratio (Turina et al. 2016). One example is a comparative study on the $\mathrm{H}^{+} / \mathrm{ATP}$ ratio of $\mathrm{F}_{\mathrm{o}} \mathrm{F}_{1}$ from Saccharomyces cerevisiae or Spinacia oleracea (Petersen et al. 2012), each of which has 10 or $14 c$ subunits (Symersky et al. 2012; Vollmar et al. 2009). The experimentally determined $\mathrm{H}^{+}$/ATP ratio was 2.9 and 3.9, respectively. The reason for the lower values than expected is unclear, although it could be attributable to the slipped rotation or a biased experimental error.

A higher $\mathrm{H}^{+}$/ATP ratio means that the $\mathrm{F}_{\mathrm{o}}$ must exert higher torque to induce ATP synthesis under a given $p m f$. The difference in the $\mathrm{H}^{+}$/ATP ratio would represent different physiological requirements. When $p m f$ is sufficiently high and stable over the life cycle of a cell, ATP production requires less protons (such as in mammalian mitochondria). However, when pmf is small and/or environmental $\Delta \mathrm{pH}$ is inverted, as it happens in alkaliphilic extremophiles (McMillan et al. 2009) or chloroplasts (Pogoryelov et al. 2005), the $F_{o}$ requires more protons to ensure constant ATP production.

The modulation of $c$-rings between organisms must be subtle, yet it is clearly essential, since it has been demonstrated, using mutagenesis, that a minor modification at the $c-c$ interface can modulate the stoichiometry of the $c$-ring (Pogoryelov et al. 2012). The rotation-mediated energy coupling mechanism of $\mathrm{F}_{\mathrm{o}} \mathrm{F}_{1}$ ATP synthase would ease the modulation of the $\mathrm{H}^{+} /$ATP ratio. This mechanism may be an advantageous feature to allow the adaptation of the ATP synthase for a variety of environmental conditions.

\section{Basic properties as a motor}

\section{Rotation assays of the $F_{1}$-ATPase}

After the establishment of the rotation assay in 1997 (Noji et al. 1997), various types of methodologies for analysis of the rotation have been reported. In all protocols, the $\alpha_{3} \beta_{3}$-ring 
of $F_{1}$ is immobilized on the coverslip surface to suppress the rotary Brownian motion of the whole body of the $F_{1}$ molecule. In most rotation assays, polyhistidine tags incorporated at the $\mathrm{N}$ terminus of the $\beta$ or $\alpha$ subunit are used to anchor the $\alpha_{3} \beta_{3}$-ring on the coverslip (Bilyard et al. 2013; Konno et al. 2006; McMillan et al. 2016; Suzuki et al. 2014; Yasuda et al. 1998). Then, a probe is attached onto the upwardly protruding part of the $\gamma$ subunit for the visualization of the rotation of the $\gamma$ subunit, of which the radius is far below optical resolution, having only a $1 \mathrm{~nm}$ radius (Abrahams et al. 1994). The first rotation assay employed a fluorescently labeled actin filament that was $0.5-5.0 \mu \mathrm{m}$ in length (Noji et al. 1997). Due to the photobleaching effect of fluorescent dye that limits the observation duration, duplexes of submicron latex beads or magnetic beads that are observable in conventional bright field imaging are now preferentially used (Fig. 3a) (Hirono-Hara et al. 2001; McMillan et al. 2016; Yasuda et al. 2001). With submicron-sized probes, the viscous friction of a probe against fluid limits the maximum rotation velocity. A typical rotation rate with these probes under saturating ATP conditions has been reported to be between 5 and 20 revolution/s and readily identified by eye (Fig. 3b, top left).

Although viscous friction-limited conditions allow us to estimate the rotational torque individual molecules generate (see the section entitled "Torque"), detailed rotation dynamics such as stepping rotation and short-lived pausing states are frequently unclear due to the slow response of the probes used. For a detailed investigation of stepping rotation, nanoparticles of size several tens of nanometers, such as gold colloids or gold nanorods, are regarded as the most useful (Spetzler et al. 2006; Ueno et al. 2010; Yasuda et al. 2001). Nanoparticles do not behave like larger probes. Their response time is much faster, thereby enabling the observation of short-lived rotational pauses of $\mathrm{F}_{1}$. In the following section, the basic rotation properties of $\mathrm{F}_{1}$ revealed by rotation assay are discussed. Due to the breadth of knowledge available, we focus on the thermophilic Bacillus PS3 ( $\left.\mathrm{TF}_{1}\right)$.

\section{Step rotation and reaction scheme}

Rotation can be resolved into stepping rotation when the intervening pause durations dominate during the recorded period of rotation observation. Stepping rotation of $\mathrm{F}_{1}$ ATPase was first observed using an actin filament under low [ATP] conditions, where ATP binding is the ratelimiting step (Fig. 3b, top right). While ATP waiting time is controllable by changing medium [ATP], other catalytic states are not readily modulated. By achieving submillisecond imaging with gold colloid $(r=40 \mathrm{~nm})$, a new rotation dwell with millisecond duration was found, revealing that the $120^{\circ}$ step can be further resolved into $80^{\circ}$ and $40^{\circ}$ substeps (Yasuda et al. 2001). Kinetic analysis with a mutant $F_{1}$ with slow catalysis revealed that the $80^{\circ}$ and $40^{\circ}$ substeps are initiated after ATP binding and hydrolysis of bound ATP, respectively (Shimabukuro et al. 2003). Thereby, the rotation dwell before each substep is referred to as binding, catalytic dwell, or pause. Several studies suggested that the ADP release occurs during binding dwell, and that the inorganic phosphate release takes place during catalytic dwell (Adachi et al. 2007; Nishizaka et al. 2004). Figure 4 shows the tentative reaction scheme of $\mathrm{TF}_{1}$. The exact timing of each reaction during pauses (at the end or at some midpoint) remains unclear.

Resolving mechanical motion into steps is a starting point to elucidate the chemomechanical coupling reactions scheme of molecular motors (Svoboda and Block 1994; Yildiz et al. 2003). Differing from architecturally simpler molecular motors like myosin and kinesin, $F_{1}$ has three reaction centers. Naturally, this results in a comparatively more challenging puzzle to solve the reaction scheme. One may understand the difficulty when considering at which angle ATP is hydrolyzed after binding. It is important to note that the $F_{1}$ has three catalytic angles in one turn and, thereby, there are, principally, three possible angles for each ATP to be hydrolyzed. A review paper that concisely introduces how this difficult puzzle has been addressed is available elsewhere (Okuno et al. 2011). Each reaction center conducts a single ATP hydrolysis event per $360^{\circ}$ $\gamma$ rotation, while the reaction phase of a catalytic site is always different from each other by $120^{\circ}$. Here, it should be stated that there is an unsettled argument on the timing of inorganic phosphate release. We propose that it occurs at $320^{\circ}$, i.e., during the third catalytic pause after binding (Watanabe et al. 2010), while Kinosita's group support a $200^{\circ}$ model (Adachi et al. 2007; Shimo-Kon et al. 2010). We are gaining strong supporting evidences for a $320^{\circ}$ model from theoretical studies and structural analysis of $\mathrm{MF}_{1}$ (Rees et al. 2012); however, it remains to be resolved in $\mathrm{TF}_{1}$. We are certain further discussion and analysis will resolve this curious facet.

Interestingly, the rotation assay on mammalian $\mathrm{F}_{1}$ (human mitochondria $\mathrm{F}_{1} ; \mathrm{hMF}_{1}$ ) showed that the $120^{\circ}$ step is resolved into three substep positions at $65^{\circ}, 25^{\circ}$, and $30^{\circ}$ (Suzuki et al. 2014). Although the reaction scheme of $\mathrm{MF}_{1}$ apparently differs from that of $\mathrm{TF}_{1}$, it can be considered to be a variation of the reaction scheme of $\mathrm{TF}_{1}$, where the hydrolysis dwell and inorganic phosphate release dwell are split into different angles. Interestingly, a recent intensive analysis using data mining techniques revealed that $\mathrm{TF}_{1}$ makes a small rotational movement during the catalytic pause that had not been previously resolved using conventional data analysis ( $\mathrm{Li}$ et al. 2015). Thus, the reaction scheme seems likely to be conserved among 


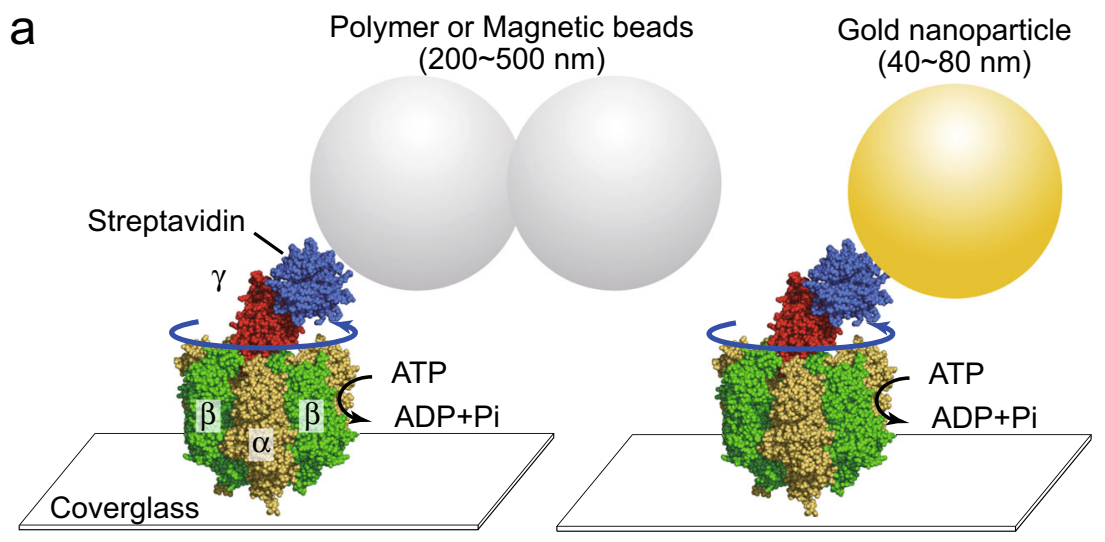

b
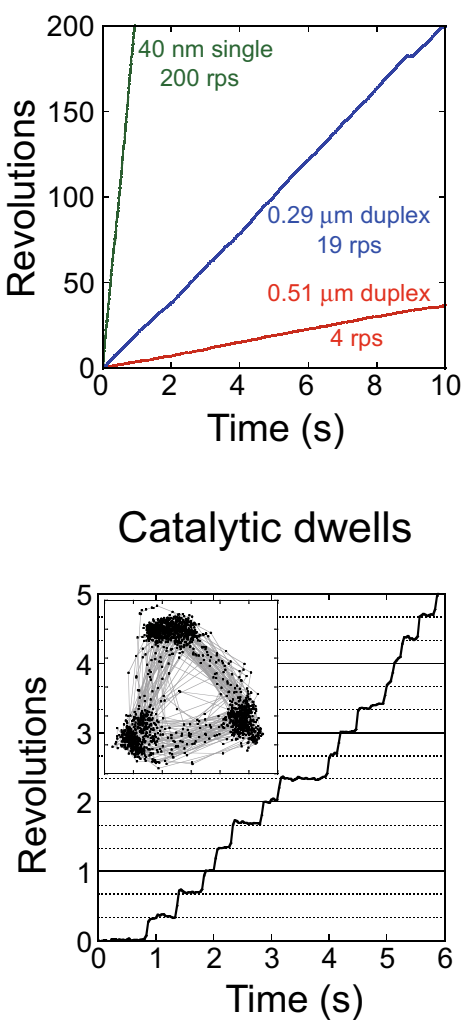

Fig. 3 Rotation assay of $F_{1}$. a A schematic image of the single-molecule rotation assay. The structural models of $\mathrm{F}_{1}$ (PDB ID: 1E79) (Gibbons et al. 2000) and streptavidin (PDB ID: 1N43) (Le Trong et al. 2003) are shown as sphere representation. The $F_{1}$-ATPase $\alpha_{3} \beta_{3}$-ring is immobilized on a glass surface, and an optical probe (fluorescently labeled actin filament, polymer beads, gold nanoparticle, gold nanorod, etc.) is attached to the $\gamma$ subunit to visualize the rotary motion of $\gamma$ subunit by an optical microscope. b Top left panel shows the time courses of rotation with various probe sizes under saturating ATP conditions (1 5 mM ATP). The top right panel represents the time course of rotation of wild-type $\mathrm{F}_{1}$ under an ATP-limiting condition (60 nM ATP),

species, although there are some variations in substep size and sequence of reaction that might reflect physiological requirements. Regarding this point, a theoretical

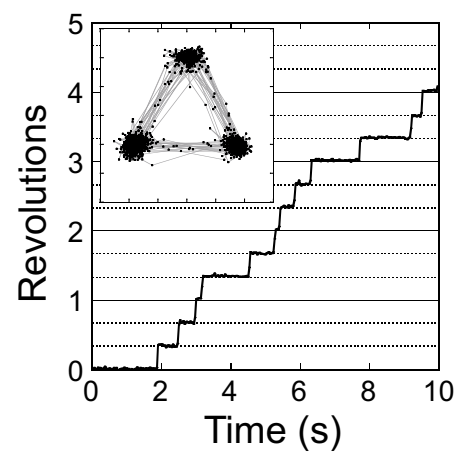

\section{Binding + Catalytic dwells}

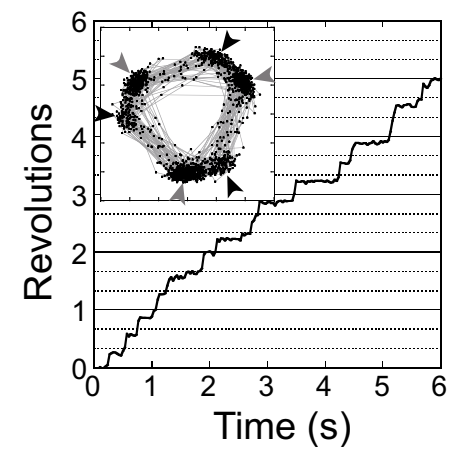

where the dwell is caused by slow ATP binding. The inset shows the trajectory of the centroid of the optical probe. The bottom left panel shows the time course of rotation of a mutant $F_{1}(\beta E 190 D)$ (Shimabukuro et al. 2003) under a saturating ATP condition ( $2 \mathrm{mM}$ ATP). Each dwell is caused by the slow catalysis by the mutant $F_{1}$. The bottom right panel shows the time course of rotation of a mutant $F_{1}$ $(\beta \mathrm{E} 190 \mathrm{D})$ around the $K_{\mathrm{m}}$ region $(2 \mu \mathrm{M}$ ATP $)$. In this condition, the $120^{\circ}$ step splits into $0^{\circ}$ and $80^{\circ}$ substeps, each intervened with a binding dwell and catalytic dwell, respectively. The black and gray arrowheads indicate the positions of ATP binding and catalytic dwell, respectively

study suggests that the position of the substeps is not critical for energy coupling efficiency (Mukherjee and Warshel 2015). 


\section{Torque}

The motion of micron-sized objects (Brownian particles) in fluid is overdamped. The acceleration and deceleration time for Brownian particles is extremely short, meaning that the Brownian motion can be treated as a Markov process. When the $\mathrm{F}_{1}$ rotates a probe in a buffered solution, the viscous friction against the rotating probe is always balanced with the torque that the $F_{1}$ generates. Thus, the torque of the $F_{1}$ can be estimated from the viscous friction determined from the probe size (i.e., the radius for a spherical probe) and observed angular velocity (Noji et al. 2001; Pänke et al. 2001; Yasuda et al. 1998). The torque of $\mathrm{TF}_{1}$ has been repeatedly reported to be around 40 pNnm (Hayashi et al. 2010; McMillan et al. 2016; Noji et al. 2001; Ueno et al. 2014). In addition, the angular velocity profile is almost constant against rotational angle. Thus, $\mathrm{TF}_{1}$ is generally considered to generate a constant $40 \mathrm{pNnm}$ of torque, irrespective of rotational angle.

One possible concern about this torque estimation is that the torque could be underestimated. This is because the viscosity of fluid near the surface is known to be higher than that in the middle. On the other hand, the surface effect could be minor, because a probe is lifted by at least $20 \mathrm{~nm}$ perpendicular to the coverslip surface by the molecules involved in the rotation assay $\left(\mathrm{F}_{1}\right.$, biotin, streptavidin, and polymer cushion on the probe surface). Furthermore, rotating particles have a slight elevation angle, being apart from the surface at the rotation edge of the probe where viscous friction is dominant. This concern was addressed, at least partially, by application of a statistical physical theory known as the fluctuation theorem. A variation of the fluctuation theorem formula adjusted to the rotation assay parameters allows for an estimation of entropy generation, i.e., the force generation only from the trajectory of probes without the consideration of physical parameters requisite for the previous method, such as fluid viscosity or size or shape of probes. A sole, but critical, requirement of this analytical method is the rapid and precise recording of the probe position. This method reconfirmed that $\mathrm{TF}_{1}$ generates a torque of $40 \mathrm{pNnm}$ (Hayashi et al. 2010).

The torque of $40 \mathrm{pNnm}$ has important implications for the energy coupling of $\mathrm{TF}_{1}$. Torque multiplied by angular displacement gives the work done by the molecular motor. In the case of the $F_{1}$, the estimated work that the $F_{1}$ does per $120^{\circ}$ rotational movement (=single turnover of ATP hydrolysis) is approximately $80 \mathrm{pNnm}$ (Yasuda et al. 1998). This is consistent with the free energy of ATP hydrolysis in a cell where $\Delta G^{\prime}{ }_{\text {ATP }}$ is $48 \mathrm{~kJ} / \mathrm{mol}$, which is equivalent to 80 $\mathrm{pNnm} /$ molecule. In addition, theoretical studies suggest that a constant torque over rotation angle is also reasonable for efficient energy coupling (Oster et al. 2000). However, recent reports challenge this view, which invite revision of the current understanding of the torque profile. Saita et al. (2015) reported that the torque profile showed three sawtooth-like peaks in a $120^{\circ}$ step. Because the observed profile is not consistent with any reported substep positions, a reasonable explanation for this conflict with previous reports is yet to be described. Sielaff (2016) reported a different torque profile. They observed that the instantaneous angular velocity of rotation probed with a gold nanorod showed a distinct peak during a $120^{\circ}$ step. Because the nanorod has a low viscous drag coefficient, it is not clear if the observed angular velocity represents the genuine torque profile of the $F_{1}$ or it includes some effect from intermolecular friction between the rotor and the stator of the $\mathrm{F}_{1}$.

We should note here that the torque could be different among species. Another model $\mathrm{F}_{1}$ well studied with rotation assay is the $\mathrm{F}_{1}$ from Escherichia coli $\left(\mathrm{EF}_{1}\right)$. Although several groups have reported different values, the reported torque of $\mathrm{EF}_{1}$ ranges from 30 to $63 \mathrm{pNnm}$ (Bilyard et al. 2013; Hornung et al. 2008; Pänke et al. 2001). Recently, an $F_{1}$ from a thermoalkaliphilic bacterium, Caldalkalibacillus thermarum, was reported to generate torque of over $50 \mathrm{pNnm}$ (McMillan et al. 2016). The $V_{1}$ motor of $V$-type ATPase from bacteria is also well studied using the rotation assay. There is a clear trend that $\mathrm{V}_{1}$-ATPases generate a lower torque than $\mathrm{F}_{1}$-ATPases: the $\mathrm{V}_{1}$ from Thermus thermophilus generates 30-35 $\mathrm{pNnm}$ and the $\mathrm{Na}^{+}$-transporting V-ATPase generates $23 \mathrm{pNnm}$ of torque, respectively (Hayashi et al. 2010; Imamura et al. 2005; Ueno et al. 2014). Although more data are required, we can see a tendency that $\mathrm{F}_{1}$ or $\mathrm{V}_{1}$ dedicated only to ATP synthesis in a cell generates a larger torque than motors working as ATPdriven ion pumps (McMillan et al. 2016).

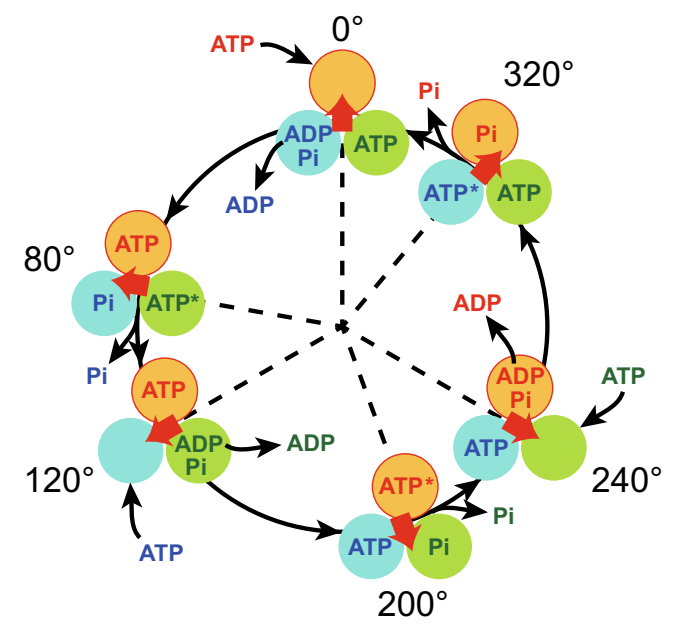

Fig. 4 Proposed chemomechanical coupling scheme of $\mathrm{TF}_{1}$. Each circle represents the chemical state of the catalytic site in each $\beta$ subunit. ATP* represents pre- or post-hydrolysis state of ATP. The central red arrow represents the orientation of the $\gamma$ subunit. $0^{\circ}$ is defined as the ATP binding angle for the catalytic site at the 12 o'clock position (orange). In this model, ATP bound at $0^{\circ}$ is cleaved into ADP and $\mathrm{Pi}$ at $200^{\circ}, \mathrm{ADP}$ dissociates at $240^{\circ}$, and then phosphate release occurs at $320^{\circ}$. Other catalytic sites (blue and green) also obey the same reaction scheme offset by $120^{\circ}$ and $240^{\circ}$ 


\section{Torque-generation step}

A milestone to meet in elucidating the mechanochemical coupling of molecular motor proteins is to identify which reaction step is principally responsible for force generation. ATP hydrolysis is resolved into at least four elementary steps: ATP binding, hydrolysis, ADP release, and inorganic phosphate release. This is a technically challenging question to address. Force measurement is a straightforward strategy. However, the catalytic reactions following ATP binding are often too fast to be resolved using force measurement as a sole strategy. Allostericity among multiple reaction sites on a motor protein also hampers the identification of the force-generation step. Another strategy that gives clues to this process is structural analysis. Interestingly, after nucleotide binding, large conformational rearrangements have been observed in the crystal structures of motor proteins.

The crystal structures of the $F_{1}$ have revealed distinct conformational difference between the $\beta$ subunits with and without bound nucleotide (Fig. 2) (Cingolani and Duncan 2011; Duncan et al. 1986; Ferguson et al. 2016; Menz et al. 2001). Several crystal structures of $\mathrm{F}_{1}$-ATPases prepared in different conditions and with different ligands have been reported so far (Abrahams et al. 1996; Braig et al. 2000; Cabezón et al. 2003; Kagawa et al. 2004; van Raaij et al. 1996). The $\beta$ subunits are the principal torque generators, and assume two distinct conformations in most structures. The $\beta$ subunit without bound nucleotide is in open conformation, while the $\beta$ subunit with bound nucleotide takes closed conformation, in which the C-terminal domain swings toward the $\gamma$ subunit (Fig. 2b) (Abrahams et al. 1994). From this observation, the ATP-binding step is proposed to be a major torque-generating step. This is quite consistent with the observation that the substep size induced by ATP binding is two times larger than that initiated after hydrolysis and inorganic phosphate release (Yasuda et al. 2001).

An alternative approach to study the force-generating step is to investigate how rotation modulates the equilibrium constant of individual reaction steps, $K_{\mathrm{E}}^{\text {reaction }}$. Standard free energy/molecule is derived from $K_{\mathrm{E}}^{\text {reaction }}$ as follows:

$\Delta G^{\mathrm{o}^{\prime}}=k_{\mathrm{B}} \operatorname{Tln} K_{\mathrm{E}}{ }^{\text {reaction }}$

where $k_{\mathrm{B}} \mathrm{T}$ represents thermal energy. Then, free energy change upon rotation, $\frac{\partial G}{\partial \theta}$ represents the magnitude of the energy that is released upon the reaction. Thus, the angledependent modulation of $K_{\mathrm{E}}^{\text {reaction }}$ is a good barometer to estimate torque generation. For this purpose, the rotation angle of the probe (a magnetic bead duplex) was controlled with magnetic tweezers, and the reaction probability was investigated as a function of rotation angle (Watanabe et al. 2012). This work revealed that $\mathrm{TF}_{1}$ exponentially tightens the affinity to ATP with rotation, while the equilibrium constant of ATP hydrolysis is slightly shifted toward hydrolysis direction. The estimated energy generation is $21-54 \mathrm{pNnm}$ for affinity change of ATP and 4-17 pNnm for equilibrium shift of hydrolysis. The reason for the range of values is that the $\gamma$ subunit must be twisted to some extent during manipulation, and the actual orientation of the $\gamma$ subunit may be overestimated. The upper and lower limits represent the estimated energy change with or without considering the elasticity of the $\gamma$ subunit. Although the exact quantification of energy release has not yet been done, this study showed that the contribution of affinity change of ATP for torque generation is over 2-fold larger than that of ATP hydrolysis.

Thus, many lines of experimental results have shown that the ATP-binding process, or more precisely the affinity change to bound ATP, is a major torque-generation step in $F_{1}$ catalysis, while the chemical cleavage of ATP has relatively minor contribution. It is less well understood how torque is generated upon ADP and inorganic phosphate release. Classic biochemical studies have shown that the inorganic phosphate uptake step is an energy-requiring step during ATP synthesis, suggesting that the inorganic phosphate-releasing step is another torque-generation step. This idea was supported by kinetic analysis on rotation of $F_{1}$ in the presence of excess inorganic phosphate (Adachi et al. 2007).

\section{Robustness}

\section{Robustness of torque transmission}

Structural mapping to explore which stator-rotor interaction is the principal method for the understanding of torque transmission. The interactions revealed by this approach have provided major insights for the elucidation of chemomechanical coupling of $F_{1}$. The $\gamma$ subunit is composed of a globular domain with an $\alpha / \beta$ fold and an antiparallel coiled-coil domain of the $\mathrm{N}$ - and $\mathrm{C}$-terminal helices. As shown in Fig. 5a, the $\gamma$ subunit is held in the $\alpha_{3} \beta_{3}$ stator ring in two positions: the upper orifice, from which the globular domain of the $\gamma$ subunit protrudes vertically, while the lower hydrophobic sleeve holds the tip of the C-terminal helix.

The stator-rotor interactions have been actively studied since 1994, when the crystal structure of $F_{1}$ became available (Abrahams et al. 1994) and many residues that impair catalysis were identified. Subsequently, the rotation assay was established, and some of these mutants were closely examined. No significant impact on the torque generation was observed using these mutants; however, kinetic parameters were affected to some extent (Hara et al. 2000; Omote et al. 1999). The robustness of the torque transmission between stator and rotor was more clearly shown by Kinosita's group. They showed that the $F_{1}$ still rotates even after being devoid of the entire $\gamma$ subunit coiled-coil axle (Fig. 5b) (Furuike et al. 2008). Although the rotation rate of $1 \mathrm{~Hz}$ was much slower than that of the wild-type $\mathrm{F}_{1}(\sim 200 \mathrm{~Hz})$ and the torque was too low to 
Fig. 5 Stator-rotor interactions within $\mathrm{F}_{1}$. a $\alpha_{\text {Empty }}-\beta_{\mathrm{ATP}}$ pair and the $\gamma$ subunit are shown as cartoon representation (PDB ID: 1E79). The blue and red circles indicate the orifice and sleeve regions in the $\alpha_{3} \beta_{3}$-ring, respectively, at which the $\gamma$ subunit is held. The helix-turnhelix $(\mathrm{HTH})$ structure of the $\beta$ subunit forming the main interface of the stator orifice is colored blue. $\mathbf{b}$ The structure of axle-less mutant $\gamma \Delta \mathrm{N} 22 \mathrm{C} 43$ (Furuike et al. 2008). The 22 residues of the $\mathrm{N}$-terminal helix and the 43 residues of the $\mathrm{C}$ terminal helix in the $\gamma$ subunit are deleted. $\mathbf{c}$ The positions of deletion in the HTH structure are shown in red (Usukura et al. 2012). d All residues of the HTH in contact with the $\gamma$ subunit are substituted with glycine or alanine (red region) (Tanigawara et al. 2012) a

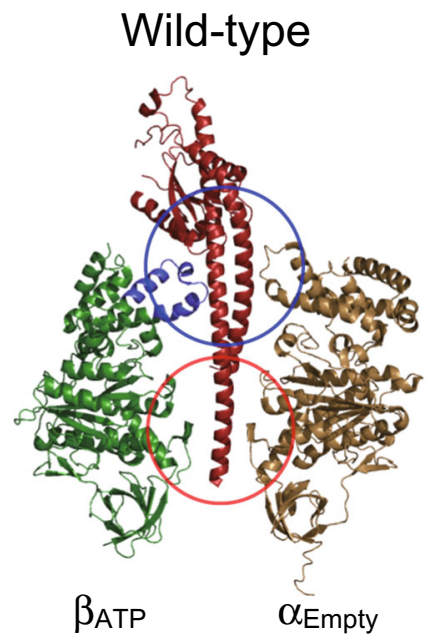

C

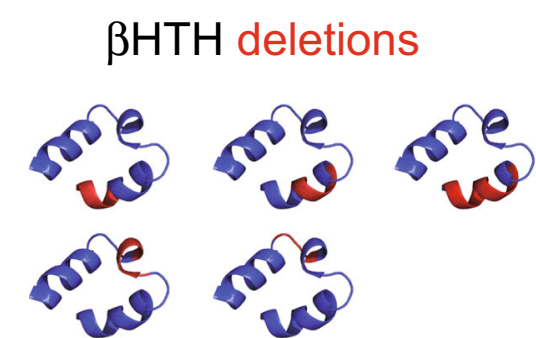

b $\gamma \Delta \mathrm{N} 22 \mathrm{C} 43$

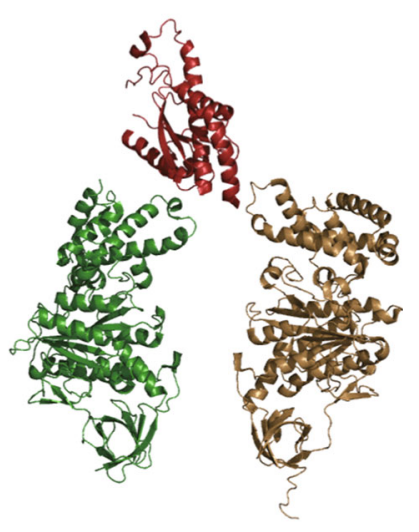

d
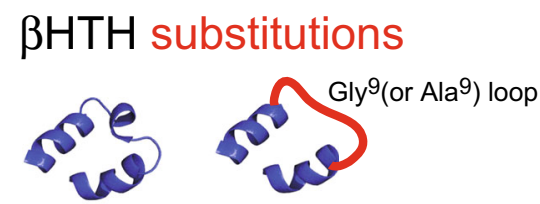

WT: ${ }^{376}$ RYKELQDIIAILGMDELSDEDKLVVHRARR ${ }^{405}$ Gly ${ }^{9}$ loop: ${ }^{376}$ RYKELQDIIA $-{ }^{386}$ Gly $^{9}{ }^{394}$ EDKLVVHRARR 405 Ala ${ }^{9}$ loop: ${ }^{376}$ RYKELQDIIA - ${ }^{386}$ Ala $^{9}{ }^{394}$ EDKLVVHRARR 405 measure, the rotation was apparently unidirectional. This finding came as a big surprise. This result means that, for the most part, the stator-rotor interaction is dispensable for unidirectional rotation. The remaining interaction in the axle-less $F_{1}$ was the upper half of the orifice interaction.

Following this, the orifice interaction for torque transmission became the focus of the following study. The helix-turnhelix (HTH) structure of the $\beta$ subunits forming the main interface of the stator orifice was investigated (Usukura et al. 2012). Figure 5a highlights the HTH structure in blue. The HTH structure is a part of the C-terminal domain of the $\beta$ subunit, which makes a large swing motion upon nucleotide binding (Abrahams et al. 1994; Masaike et al. 2008). The HTH structure was shortened by the deletion of one or two turns (Fig. 5c). The resultant mutant $F_{1}$ still showed clear rotation (Usukura et al. 2012). The orifice interaction was comprehensively shown to be dispensable. This point was confirmed in another mutagenesis study where all residues of the HTH in contact with the $\gamma$ subunit were substituted with alanine or glycine residues (Fig. 5d) (Tanigawara et al. 2012). Surprisingly, the mutant $\mathrm{F}_{1}$ with Ala substitutions generated a torque comparable with the wild-type $\mathrm{F}_{1}$ values ( $97 \%$ of the wild-type), suggesting that specific interactions at the orifice interface are not entirely requisite for full torque transmission. The mutant $F_{1}$ with Gly substitutions replacing 22 residues of the HTH still rotated; however, the torque was reduced to $60 \%$ of the wild-type. Molecular dynamics simulations suggested that the Gly mutant completely lost the rigidity of the HTH structure, while the Ala mutant still keeps the structure of the HTH (Tanigawara et al. 2012). These findings suggest that full torque transmission at the orifice interface does not require specific interaction, but the rigidity of the HTH structure. It is highly likely that the HTH structure works as a "push rod" for the $\beta$ subunit to induce unidirectional rotation.

Thus, the series of extensive mutation studies revealed that all parts of the stator-rotor interface are dispensable, and that the torque transmission mechanism is far more robust than previously thought. The mutagenesis studies suggest that the orifice interaction is responsible for roughly half of the torque transmission. The sleeve interaction probably transmits the other half of the torque. The remaining issue to be explored is how the $\alpha_{3} \beta_{3}$-ring generates torque at the sleeve interaction. From the crystal structures, it is not clear what type of conformational change is responsible for the torque transmission at the sleeve interaction.

\section{Robustness of allostericity: from dictator model to professor model}

The robustness of torque transmission also implies the robustness of the cooperativity among the three catalytic reaction centers. The rotation of axles-less $F_{1}$ brought surprise not only in the context of the torque transmission, but also in that of the allostericity of $F_{1}$. This is because, at the time, the so-called $\gamma$ - 
dictator model was prevailing. This model assumes that the interaction with the $\gamma$ subunit fully controls the timing of catalysis in the $\beta$ subunits. Another underlying reason is a biochemical study which showed that the isolated $\alpha_{3} \beta_{3}$-ring did not show obvious cooperativity among catalytic sites (Kaibara et al. 1996). The following experiments that reinforced the $\gamma$-dictator model were demonstrations that ATP synthesis is possible upon forced reverse rotation of $F_{1}$ (Itoh et al. 2004; Rondelez et al. 2005). These experiments showed that the equilibrium constant of ATP hydrolysis is over $10^{5}$ at room temperature in aqueous solution, and can be easily modulated to be less than 1 by controlling the angle orientation of the $\gamma$ subunit.

The aforementioned mutagenesis studies on the stator-rotor interface imply that there is no indispensable stator-rotor interaction to control cooperative catalysis for unidirectional rotation. To visualize sequential catalysis accompanying the power-stroke motion of the $\beta$ subunit in the isolated stator complex (the $\alpha_{3} \beta_{3}$ subcomplex), high-speed atomic force microscopy (HS-AFM) was employed (Fig. 6). This imaging method allowed visualization of the structural change of biomolecules at work in aqueous solution at over 10 frames per second with subnanometer spatial resolution (Kodera and Ando 2014; Uchihashi et al. 2011). The isolated $\alpha_{3} \beta_{3}$-ring was covalently immobilized on mica surface for HS-AFM observation. In agreement with the crystal structures of $F_{1}$, when the unhydrolyzable nucleotide (adenylylimidodiphosphate, AMP-PNP) was added to the solution, the $\alpha_{3} \beta_{3}$-ring adopts a CCO state. Two of the three $\beta$ subunits take the closed form $(\mathrm{C})$, where the $\mathrm{C}$-terminal domain swings down, while the third took the open form $(\mathrm{O})$, with the Cterminal domain protruding vertically (Fig. 6b). Dynamics were also observed in the presence of ATP. The $\alpha_{3} \beta_{3}$-ring rotationally propagated the $\mathrm{CCO}$ state in a counterclockwise direction similar to the $\gamma$ rotation; when the $\beta$ in the $\mathrm{C}$ state at the clockwise side of a CC pair makes a conformational C-to$\mathrm{O}$ transition, the $\beta$ in the $\mathrm{O}$ state makes an $\mathrm{O}$-to- $\mathrm{C}$ transition (Fig. 6c, d). The rate of the transition $\left(1.5 \mathrm{~s}^{-1}\right.$ at $2 \mu \mathrm{M}$ ATP) was consistent with the rate of ATP hydrolysis measured in biochemical analysis $\left(1.6 \mathrm{~s}^{-1}\right.$ at $2 \mu \mathrm{M}$ ATP). Thus, it was clearly shown that three $\beta$ subunits undergo sequential power-stroke conformational transitions coupled with ATP hydrolysis.

This is the decisive evidence that the $\alpha_{3} \beta_{3}$-ring has intrinsic cooperativity among the three catalytic sites, and that the $\gamma$ subunit dictator model is likely incorrect for $F_{1}$. However, the rate of the observed conformational transition, $1-4 \mathrm{~Hz}$, was remarkably slower than with the $\gamma$ subunit, being 100 $200 \mathrm{~Hz}$. In addition, the occasional back-step was also observed, suggesting that the cooperativity does not reach perfection without the $\gamma$ subunit. Therefore, we propose that the "professor" model might be a better representation for the allostery of $F_{1}$ than "intrinsic cooperativity". Here, "professor" means an existence that is not indispensable but enhances the activity of a system or organization. ${ }^{1}$

A question that then arose based on this finding was "can an exogenous rod-shaped protein rotate in the $\alpha_{3} \beta_{3}$-ring?" To test this, attempts were then made to insert the FliJ protein into the $\alpha_{3} \beta_{3}$-ring (Fig. 7) (Baba et al. 2016). FliJ is a component of the bacterial flagellar type III export system and its function has not yet been described (Ibuki et al. 2011). FliJ also has an antiparallel coiled-coil with a similar length to the $\gamma$ subunit; therefore, it has a similar morphology. Importantly, there is no homology in the primary structure between FliJ and the $\gamma$ subunit (Fig. 7a), and the surface charge density is opposite to each other. FliJ has a net negative charge, while the $\gamma$ subunit has more positively charged residues. The resultant hybrid motor showed unidirectional rotation in the counterclockwise direction (Fig. 7b, right, cyan lines). However, torque was only $10 \%$ of the torque of the wild-type $\mathrm{F}_{1}$ (Baba et al. 2016). FliJ was also inserted into the stator ring from a VATPase to ensure that the robustness of torque transmission is conserved between $F_{1}$ and $V_{1}$ molecular motors. Surprisingly, the $\mathrm{V}_{1}$-FliJ hybrid motor showed active unidirectional rotation with a measured torque that is comparable to that of the native V-ATPase (Baba et al. 2016). This is probably because the shape of the rotor from the $\mathrm{V}_{1}$-ATPase is more similar to FliJ than the $\gamma$ subunit of $F_{1}$ (Fig. 7c). These findings strongly suggest that the torque transmission in F- and V-type ATPases do not require any residue-specific interaction between stator and rotor, but do require gross special/shape matching for efficient torque transmission.

\section{Robustness of catalytic power}

Robustness was also revealed in the catalytic power of the $F_{1}$. Although the $F_{1}$ is unique in its high reversibility and efficiency of the chemomechanical coupling, the $F_{1}$ shares common structural features of the catalytic site with other nucleotide-driven molecular machines. One of the most well conserved structural features is the phosphate-binding motif, the so-called p-loop, or the Walker motif B that is found in most NTPases. The p-loop motif has a GXXXXGKT/S sequence (Walker et al. 1982). The seventh lysine, the p-loop lysine, is the most conserved, and is the gold standard target to knock out the catalytic activity of any described NTPase. In support of this, when substituted with another non-charged residue such as alanine, the catalytic activity reduces to undetectable levels in biochemical analysis (Senior and Al-Shawi 1992; Yagi et al. 2009). Substitution of the p-loop lysine in $\mathrm{F}_{1}$ is known to abolish all ATPase activity (Hanson and Whiteheart 2005).

\footnotetext{
${ }^{1}$ Of course, this is a self-deprecating analogy. It could only be the case in Japan.
} 
Fig. 6 High-speed atomic force microscopy (HS-AFM) imaging of the isolated $\alpha_{3} \beta_{3}$-ring. a Averaged AFM image without nucleotide when observed from the $\mathrm{C}$-terminal side. The red arrows indicate $\beta$ subunits showing an open conformation (Uchihashi et al. 2011). b Averaged AFM image at $1 \mathrm{mM}$ AMP-PNP. The blue arrows indicate the $\beta$ subunits showing a closed conformation. $\mathbf{c}$

Successive HS-AFM images showing conformational change of the $\beta$ subunits at $2 \mu \mathrm{M}$ ATP (left-to-right and top-to-bottom). The red circles indicate the highest pixel in each image. The frame rate of imaging is $12.5 / \mathrm{s}$. d Time course of the cumulated number of counterclockwise shifts of the CCO state. The black circles, crosses, and pluses represent $\mathrm{CCO}, \mathrm{COO}$, and other irregular states, respectively a

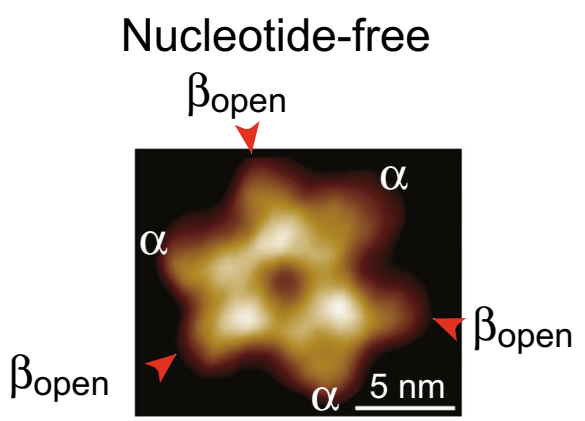

C

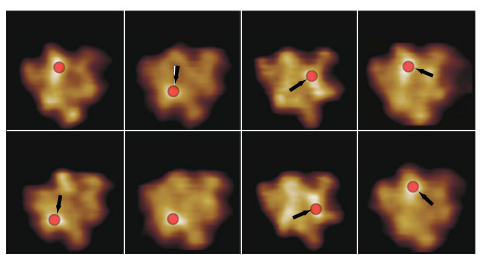

b

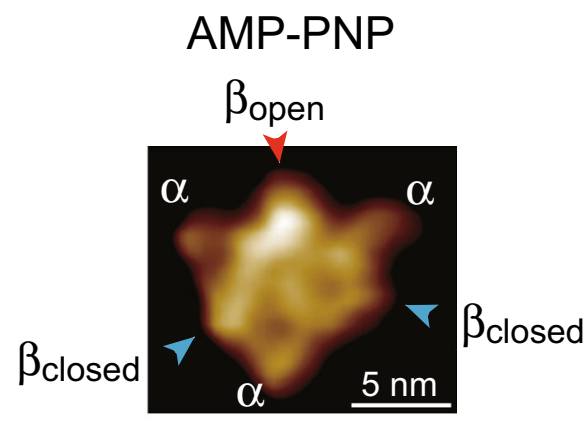

d

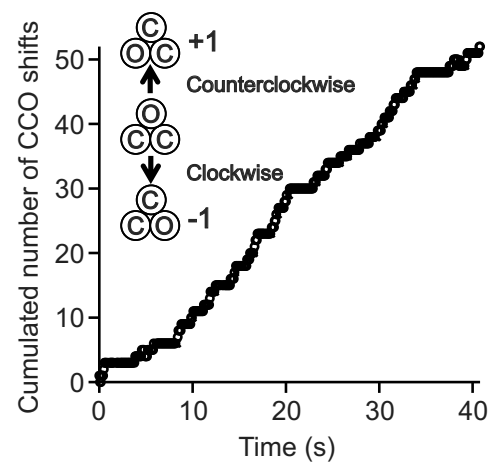

The $\mathrm{F}_{1}$-ATPase has another well-conserved charged residue directly associating with bound ATP. Many of the NTPase proteins share a glutamic acid residue that seems to associate with bound nucleotide via a coordinated water molecule at the distal end of the $\gamma$-phosphate. This glutamic acid residue is critically important for the catalysis of $\mathrm{F}_{1}$ (Shimabukuro et al. 2003). When substituted with an aspartic acid, the rate of $F_{1}$ catalysis is reduced by over 100 -fold. When substituted with non-charged residue, the catalytic activity was undetectable in biochemical assays (Amano et al. 1994, 1999). Previously, it was proposed that the glutamic acid residue activates the coordinated water by removing a proton and induces the in-line attachment of the activated water to the $\gamma$-phosphate of ATP. Therefore, the glutamic acid residue was termed a "general base". However, a recent theoretical study revised the working mechanism of the glutamic acid residue, showing that hydrolysis at the $\gamma$-phosphate spontaneously occurs before the protonation of the glutamic acid residue (Hayashi et al. 2012). The proposed role of this residue is to build a hydrogen network for the enhancement of proton transfer, the kinetically rate-determining step of ATP hydrolysis.

Another common feature of the catalytic site of $F_{1}$ with other NTPase proteins is the catalytic arginine residue, the so-called arginine finger. The arginine finger was first found in a G-protein activating protein (GAP), and was proposed to be a catalytic switch to initiate the hydrolysis of GTP bound on G-protein (Ahmadian et al. 1997). The corresponding arginine residues were widely found in other NTPases, including RecA type proteins, of which $\mathrm{F}_{1}$ is a more distal family member, and to the $\mathrm{AAA}^{+}$family proteins (Hanson and Whiteheart 2005). The arginine finger of $F_{1}$ resides on the $\alpha$ subunit. Many lines of experimental research have shown that the arginine finger has a crucial role in the catalysis of $\mathrm{F}_{1}$ (Hatch et al. 1995; Komoriya et al. 2012).

These three charged residues of $F_{1}$ were identified as catalytically critical residues in theoretical studies (Dittrich et al. 2004). Mutagenesis studies also showed that, when these residues are substituted with non-charged residues such as alanine, the catalytic power is abolished (Senior and Al-Shawi 1992; Yagi et al. 2009). Alanine mutants at these residues were re-investigated in rotation assay systems to confirm their critical role in catalysis (Watanabe et al. 2014). Against all expectations, all of the mutants showed unidirectional rotation. The rotational rate was significantly lower by a factor of $10^{3}$ to $10^{4}$. Such a low catalysis rate is not detectable in biochemical analysis. While the large impact of the mutation on catalysis is consistent with previous reports and theoretical analysis, the robustness of the catalytic power is unexpected. This finding reveals that the catalytic site architecture is designed to be quite robust against both point mutations and possibly more severe mutagenic perturbations.

Interestingly, all of the alanine mutants had a lower torque than the wild-type enzyme. The most remarkable impact was found in the alanine substitution at p-loop lysine, causing a $75 \%$ reduction of torque. Arginine finger and catalytic glutamic acid residue mutants also resulted in a torque 
a

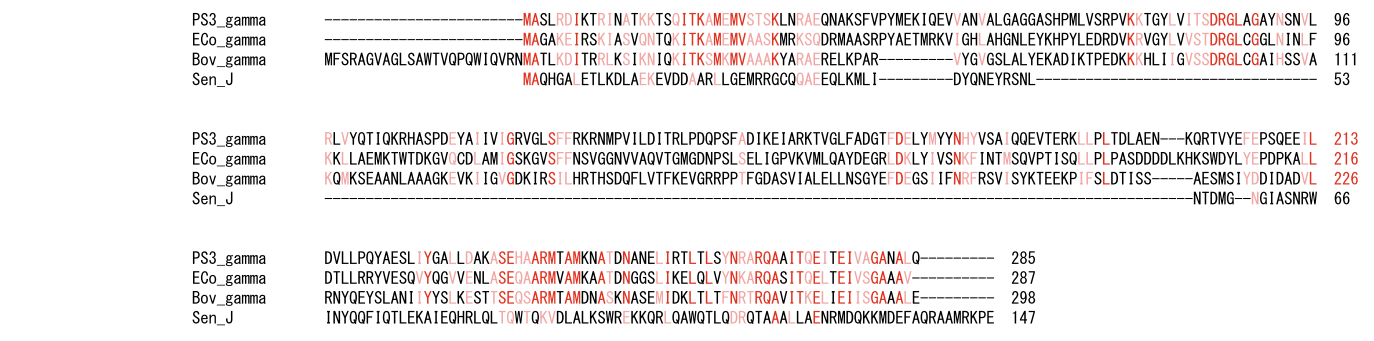

b

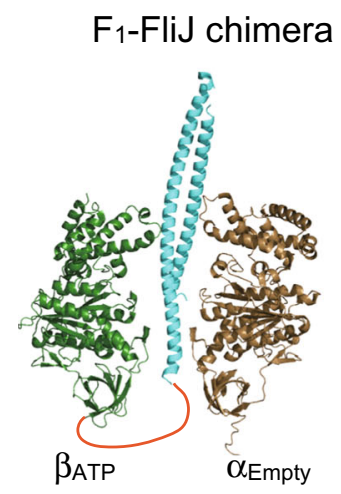

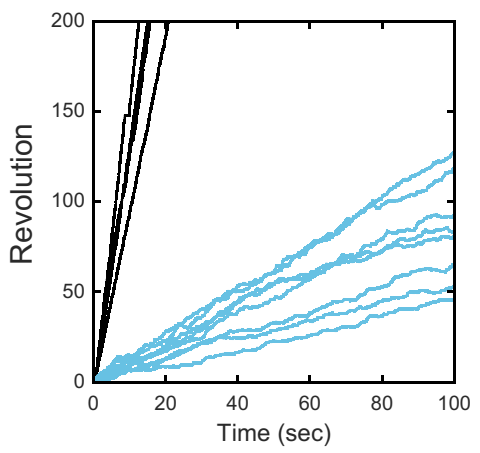

C
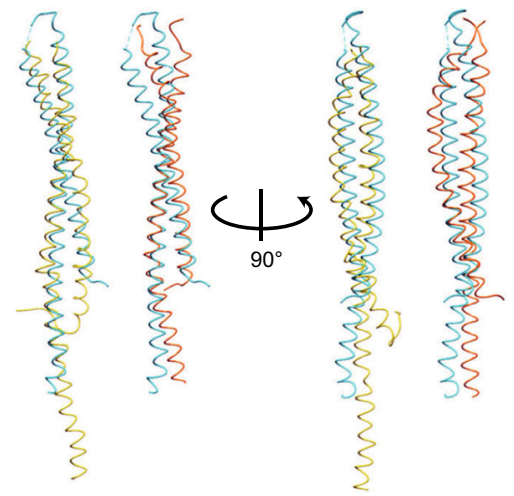

Fig. 7 Rotation of $F_{1}-$ FliJ chimera. a Sequence alignment of FliJ and $F_{1}$ $\gamma$ subunit. The amino acid sequences of $\mathrm{F}_{1}-\gamma$ from thermophilic Bacillus PS3 (PS3- $\gamma$ ), Escherichia coli (Eco- $\gamma$ ), and bovine mitochondria (Bov- $\gamma$ ) were aligned using ClustalW. The FliJ sequence (Sen-J, PDB ID: 3AJW) and Bov- $\gamma$ (PDB ID: 1E79) were structurally aligned using the MATRAS server due to the low sequence similarity between them (Baba et al. 2016). The conserved residues are highlighted in red (identical) or pink (strong similarity). b Left panel shows the structure model of $F_{1}$-FliJ chimera. $\alpha_{\text {Empty }}-\beta_{\text {ATP }}$ pair (PDB ID: 1E79) and FliJ (cyan, PDB ID:

3AJW) are shown as cartoon representations. The linker portion is represented by an orange line. The right panel shows the time courses of rotation of $\mathrm{F}_{1}$ (black lines) and $\mathrm{F}_{1}$-FliJ chimera (cyan lines). c Structural alignment of FliJ and $\mathrm{V}_{1}-\mathrm{D}$ or $\mathrm{F}_{1}-\gamma$ subunit. FliJ (cyan, PDB ID: $3 \mathrm{AJW}$ ) was superimposed on the $\mathrm{D}$ subunit of $\mathrm{V}_{1}$ (yellow, PDB ID: $3 \mathrm{~W} 3 \mathrm{~A}$ ) or the $\gamma$ subunit of $\mathrm{F}_{1}$ (orange, PDB ID: 4XD7) using the MATRAS server. Root-mean-square deviations (RMSDs) between FliJ and the $\mathrm{D}$ subunit of $\mathrm{V}_{1}$ or $\gamma$ subunit of $\mathrm{F}_{1}$ are $3.2 \AA$ or $4.4 \AA$, respectively

Fig. 8 Two-step ATP binding model. The upper and lower panels show the conformational states of the $\beta$ subunit and the chemical states in the binding pocket during ATP binding and subsequent torque generation. The ATP-binding process consists of two steps (first docking and second induced fit). The first docking process is triggered by the recognition of the base portion of ATP, and the subsequent induced-fit process is triggered by the recognition of the phosphate portion, which contributes to the torque generation (Arai et al. 2014)
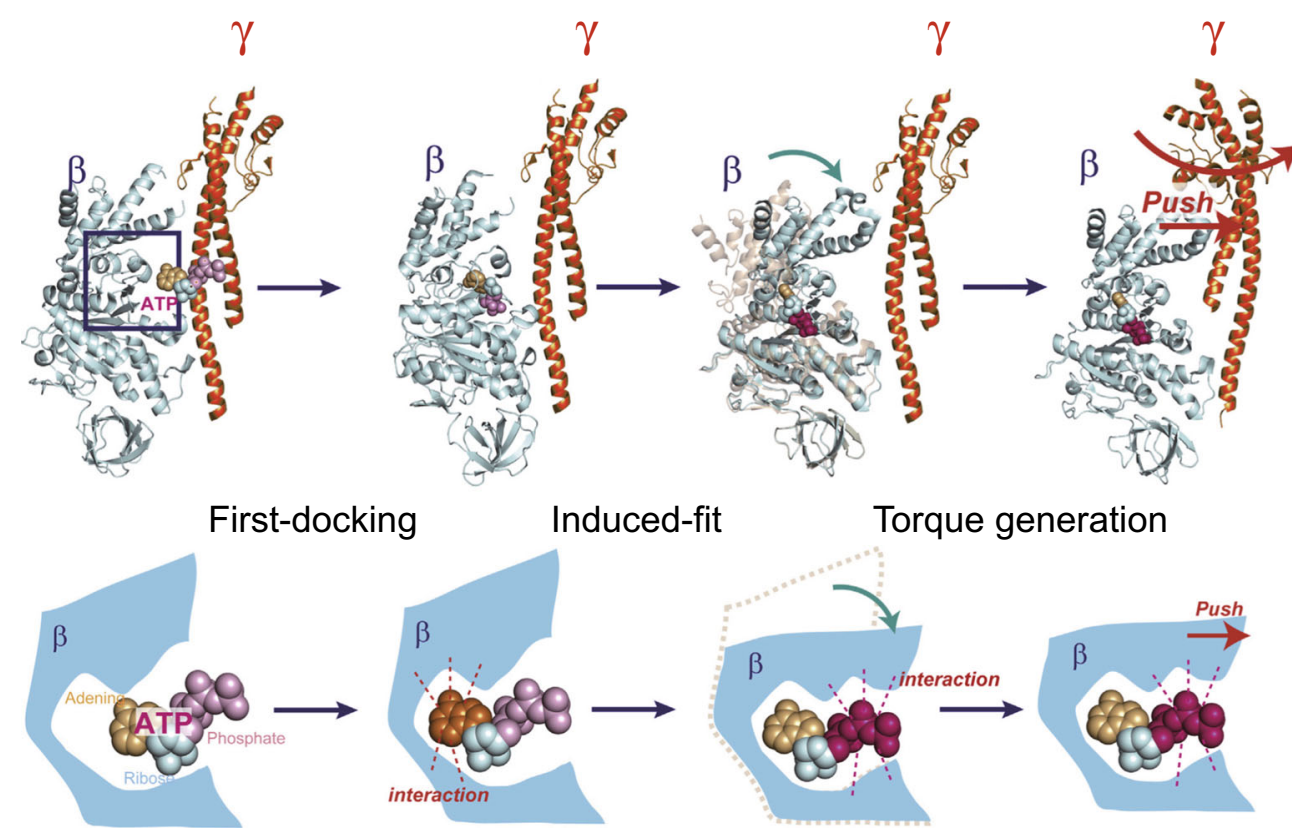
reduction of 50\%. This is in contrast to the impact of chemical modification of ATP on torque generation (Arai et al. 2014). The $\mathrm{F}_{1}$ has broad nucleotide specificity, hydrolyzing other nucleotides: GTP, CTP, and UTP, albeit with changes in the catalysis rate. Even when the base structure is removed, the $\mathrm{F}_{1}$ hydrolyzes base-free nucleotide, inducing $\gamma$ subunit rotation. The impact of the modification or depletion of base in kinetics was remarkable; the binding constants of UTP and base-free nucleotide were $10^{3}$ and $10^{6}$ times slower than ATP. However, rotational torque was retained at a comparable level to that of ATP-driven rotation. This means that the interaction with the base structure of nucleotide is critical for the enhancement of binding, but not for torque generation. This is in contrast to the impact of mutation at the phosphate-binding residues, which largely impaired the kinetic power as well as torque generation (Arai et al. 2014; Watanabe et al. 2014).

As previously mentioned, the affinity change to bound ATP is a major torque-generation step. Considering these findings, we propose a two-step ATP binding model: the first substrate docking process and the following induced-fit process accompanying affinity change. The base structure of ATP is responsible for the first substrate recognition process, but it is not involved in the induced-fit process, while the binding of phosphate to the catalytic residues is principally responsible for the induced-fit process. A schematic view is depicted in Fig. 8. Considering the common structural features in the catalytic site, the phosphate-induced power-stroke mechanism is highly likely to be conserved among other molecular machines.

\section{Perspective}

During the past two decades, single-molecule rotation assays have uncovered many aspects of the $\mathrm{F}_{1}$-ATPase catalytic mechanism. Basic properties of the $F_{1}$ as a rotary motor, such as step size, rotational velocity, torque, and kinetic parameters of rotation, were all clearly revealed. Most parts of the reaction scheme have also been elucidated in combination with knowledge from structural analysis and biochemical studies. Theoretical studies have also been exceedingly helpful in contributing to the elucidation of the molecular mechanism of the $\mathrm{F}_{1}$. The torque-generation mechanism is also partly revealed. However, one must note that analysis and interpretation do not guarantee our understanding. A practical test would be an engineering approach where we design, build, and test chimeras of molecular motors. A hybrid motor of $F_{1}$ and FliJ is an initial step toward the engineering approach (Pogoryelov et al. 2012), but we are still clearly at the preliminary stages of this approach. Theoretical toolboxes for de novo designing of proteins are growing rapidly. By combining the rational designing methods with directed evolution technology, we will soon be able to build de novo molecular motors. The feedback loop between analytical approaches represented by single- molecule rotation assays, and structural analysis, and engineering approaches will further our understanding of mechanochemical coupling mechanisms and design principles in naturally occurring protein machinery.

Acknowledgements This work was supported in part by Core Research for Evolutional Science and Technology (CREST) from Japan Science and Technology Agency (JST), and Grant-in-Aids for Scientific Research (nos. 18074005 and 22247025 to H.N.) from the Ministry of Education, Culture, Sports, Science and Technology of Japan. We thank all members of our laboratory.

\section{Compliance with ethical standards}

Conflict of interest Hiroyuki Noji declares that he has no conflicts of interest. Hiroshi Ueno declares that he has no conflicts of interest. Duncan G. G. McMillan declares that he has no conflicts of interest.

Ethical approval This article does not contain any studies with human participants or animals performed by any of the authors.

Open Access This article is distributed under the terms of the Creative Commons Attribution 4.0 International License (http:// creativecommons.org/licenses/by/4.0/), which permits unrestricted use, distribution, and reproduction in any medium, provided you give appropriate credit to the original author(s) and the source, provide a link to the Creative Commons license, and indicate if changes were made.

\section{References}

Abrahams JP, Leslie AG, Lutter R, Walker JE (1994) Structure at 2.8 a resolution of F1-ATPase from bovine heart mitochondria. Nature 370:621-628. doi:10.1038/370621a0

Abrahams JP, Buchanan SK, Van Raaij MJ, Fearnley IM, Leslie AG, Walker JE (1996) The structure of bovine F1-ATPase complexed with the peptide antibiotic efrapeptin. Proc Natl Acad Sci U S A 93:9420-9424

Adachi K, Oiwa K, Nishizaka T, Furuike S, Noji H, Itoh H, Yoshida M, Kinosita K (2007) Coupling of rotation and catalysis in F(1)-ATPase revealed by single-molecule imaging and manipulation. Cell 130: 309-321. doi:10.1016/j.cell.2007.05.020

Ahmad Z, Senior AE (2005) Involvement of ATP synthase residues $\alpha$ Arg-376, $\beta$ Arg-182, and $\beta$ Lys-155 in Pi binding. FEBS Lett 579:523-528. doi:10.1016/j.febslet.2004.12.022

Ahmadian MR, Stege P, Scheffzek K, Wittinghofer A (1997) Confirmation of the arginine-finger hypothesis for the GAP-stimulated GTP-hydrolysis reaction of Ras. Nat Struct Biol 4:686-689

Allegretti M, Klusch N, Mills DJ, Vonck J, Kühlbrandt W, Davies KM (2015) Horizontal membrane-intrinsic $\alpha$-helices in the stator $a$-subunit of an Ftype ATP synthase. Nature 521:237-240. doi:10.1038/nature14185

Amano T, Tozawa K, Yoshida M, Murakami H (1994) Spatial precision of a catalytic carboxylate of F1-ATPase $\beta$ subunit probed by introducing different carboxylate-containing side chains. FEBS Lett 348: 93-98. doi:10.1016/0014-5793(94)00588-5

Amano T, Matsui T, Muneyuki E, Noji H, Hara K, Yoshida M, Hisabori T (1999) $\alpha 3 \beta 3 \gamma$ complex of F1-ATPase from thermophilic Bacillus PS3 can maintain steady-state ATP hydrolysis activity depending on the number of non-catalytic sites. Biochem J 343(Pt 1):135-138

Arai HC, Yukawa A, Iwatate RJ, Kamiya M, Watanabe R, Urano Y, Noji H (2014) Torque generation mechanism of F1-ATPase upon NTP binding. Biophys J 107:156-164. doi:10.1016/j.bpj.2014.05.016 
Baba M, Iwamoto K, Iino R, Ueno H, Hara M, Nakanishi A, Kishikawa JI, Noji H, Yokoyama K (2016) Rotation of artificial rotor axles in rotary molecular motors. Proc Natl Acad Sci U S A 113:11214 11219. doi:10.1073/pnas. 1605640113

Ballhausen B, Altendorf K, Deckers-Hebestreit G (2009) Constant c10 ring stoichiometry in the Escherichia coli ATP synthase analyzed by cross-linking. J Bacteriol 191:2400-2404. doi:10.1128/JB. 01390-08

Bilyard T, Nakanishi-Matsui M, Steel BC, Pilizota T, Nord AL, Hosokawa H, Futai M, Berry RM (2013) High-resolution singlemolecule characterization of the enzymatic states in Escherichia coli F1-ATPase. Philos Trans R Soc Lond Ser B Biol Sci 368:20120023. doi:10.1098/rstb.2012.0023

Böttcher B, Gräber P (2000) The structure of the H(+)-ATP synthase from chloroplasts and its subcomplexes as revealed by electron microscopy. Biochim Biophys Acta 1458:404-416

Braig K, Menz RI, Montgomery MG, Leslie AG, Walker JE (2000) Structure of bovine mitochondrial F(1)-ATPase inhibited by $\operatorname{mg}(2+)$ ADP and aluminium fluoride. Structure 8:567-573

Cabezón E, Montgomery MG, Leslie AG, Walker JE (2003) The structure of bovine F1-ATPase in complex with its regulatory protein IF1. Nat Struct Mol Biol 10:744-750. doi:10.1038/nsb966

Cingolani G, Duncan TM (2011) Structure of the ATP synthase catalytic complex $(\mathrm{F}(1))$ from Escherichia coli in an autoinhibited conformation. Nat Struct Mol Biol 18:701-707. doi:10.1038/nsmb.2058

Dimroth P, von Ballmoos C, Meier T (2006) Catalytic and mechanical cycles in F-ATP synthases. Fourth in the Cycles Review Series. EMBO Rep 7:276-282. doi:10.1038/sj.embor.7400646

Dittrich M, Hayashi S, Schulten K (2004) ATP hydrolysis in the betaTP and betaDP catalytic sites of F1-ATPase. Biophys J 87:2954-2967. doi:10.1529/biophysj.104.046128

Duncan TM, Parsonage D, Senior AE (1986) Structure of the nucleotidebinding domain in the $\beta$-subunit of Escherichia coli F1-ATPase. FEBS Lett 208:1-6

Dunn SD, Chandler J (1998) Characterization of a b2 $\delta$ complex from Escherichia coli ATP synthase. J Biol Chem 273:8646-8651

Düser MG, Zarrabi N, Cipriano DJ, Ernst S, Glick GD, Dunn SD, Börsch $\mathrm{M}(2009) 36^{\circ}$ step size of proton-driven c-ring rotation in FoF1-ATP synthase. EMBO J 28:2689-2696. doi:10.1038/emboj.2009.213

Feniouk BA, Yoshida M (2008) Regulatory mechanisms of protontranslocating $\mathrm{F}(\mathrm{O}) \mathrm{F}(1)-\mathrm{ATP}$ synthase. Results Probl Cell Differ 45: 279-308. doi:10.1007/400 2007043

Feniouk BA, Kato-Yamada Y, Yoshida M, Suzuki T (2010) Conformational transitions of subunit $\varepsilon$ in ATP synthase from thermophilic Bacillus PS3. Biophys J 98:434-442. doi:10.1016/j.bpj. 2009.10.023

Ferguson SA, Cook GM, Montgomery MG, Leslie AGW, Walker JE (2016) Regulation of the thermoalkaliphilic F1-ATPase from Caldalkalibacillus thermarum. Proc Natl Acad Sci U S A 113: 10860-10865. doi:10.1073/pnas.1612035113

Furuike S, Hossain MD, Maki K, Adachi K, Suzuki T, Kohori A, Itoh H, Yoshida M, Kinosita K (2008) Axle-less F1-ATPase rotates in the correct direction. Science (New York, NY) 319:955-958. doi:10. 1126/science. 1151343

Gibbons C, Montgomery MG, Leslie AG, Walker JE (2000) The structure of the central stalk in bovine $\mathrm{F}(1)$-ATPase at $2.4 \AA$ resolution. Nat Struct Mol Biol 7:1055-1061. doi:10.1038/80981

Hanson PI, Whiteheart SW (2005) AAA+ proteins: have engine, will work. Nat Rev Mol Cell Biol 6:519-529. doi:10.1038/nrm1684

Hara KY, Noji H, Bald D, Yasuda R, Kinosita K Jr, Yoshida M (2000) The role of the DELSEED motif of the $\beta$ subunit in rotation of F1ATPase. J Biol Chem 275:14260-14263

Hara KY, Kato-Yamada Y, Kikuchi Y, Hisabori T, Yoshida M (2001) The role of the $\beta$ DELSEED motif of F1-ATPase: propagation of the inhibitory effect of the $\varepsilon$ subunit. J Biol Chem 276:23969-23973. doi:10.1074/jbc.M009303200
Hatch LP, Cox GB, Howitt SM (1995) The essential arginine residue at position 210 in the $a$ subunit of the Escherichia coli ATP synthase can be transferred to position 252 with partial retention of activity. J Biol Chem 270:29407-29412

Hayashi K, Ueno H, Iino R, Noji H (2010) Fluctuation theorem applied to F1-ATPase. Phys Rev Lett 104:218103

Hayashi S, Ueno H, Shaikh AR, Umemura M, Kamiya M, Ito Y, Ikeguchi M, Komoriya Y, Ino R, Noji H (2012) Molecular mechanism of ATP hydrolysis in F1-ATPase revealed by molecular simulations and single-molecule observations. J Am Chem Soc 134:84478454. doi:10.1021/ja211027m

Hirono-Hara Y, Noji H, Nishiura M, Muneyuki R, Hara KY, Yasuda R, Kinosita K, Yoshida M (2001) Pause and rotation of F(1)-ATPase during catalysis. Proc Natl Acad Sci U S A 98:13649-13654. doi: 10.1073/pnas.241365698

Hornung T, Ishmukhametov R, Spetzler D, Martin J, Frasch WD (2008) Determination of torque generation from the power stroke of Escherichia coli F1-ATPase. Biochim Biophys Acta 1777:579582. doi:10.1016/j.bbabio.2008.04.016

Ibuki T, Imada K, Minamino T, Kato T, Miyata T, Namba K (2011) Common architecture of the flagellar type III protein export apparatus and F- and V-type ATPases. Nat Struct Mol Biol 18:277-282. doi:10.1038/nsmb.1977

Imamura $\mathrm{H}$, Takeda $\mathrm{M}$, Funamoto $\mathrm{S}$, Shimabukuro K, Yoshida $\mathrm{M}$, Yokoyama K (2005) Rotation scheme of V1-motor is different from that of F1-motor. Proc Natl Acad Sci U S A 102:17929-17933. doi: 10.1073/pnas.0507764102

Ishmukhametov R, Hornung T, Spetzler D, Frasch WD (2010) Direct observation of stepped proteolipid ring rotation in E. coli $\mathrm{F}(\mathrm{o}) \mathrm{F}(1)-\mathrm{ATP}$ synthase. EMBO J 29:3911-3923. doi:10.1038/emboj.2010.259

Itoh H, Takahashi A, Adachi K, Noji H, Yasuda R, Yoshida M, Kinosita K (2004) Mechanically driven ATP synthesis by F1-ATPase. Nature 427:465-468. doi:10.1038/nature02212

Jackson JB (2016) Natural pH gradients in hydrothermal alkali vents were unlikely to have played a role in the origin of life. J Mol Evol 83:1-11. doi:10.1007/s00239-016-9756-6

Junge W, Nelson N (2005) Nature's rotary electromotors. Science (New York, NY) 308:642-644

Junge W, Lill H, Engelbrecht S (1997) ATP synthase: an electrochemical transducer with rotatory mechanics. Trends Biochem Sci 22:420-423

Junge W, Sielaff H, Engelbrecht S (2009) Torque generation and elastic power transmission in the rotary $\mathrm{F}(\mathrm{O}) \mathrm{F}(1)-\mathrm{ATPase}$. Nature 459: 364-370. doi:10.1038/nature08145

Kagawa R, Montgomery MG, Braig K, Leslie AG, Walker JE (2004) The structure of bovine F1-ATPase inhibited by ADP and beryllium fluoride. EMBO J 23:2734-2744. doi:10.1038/sj.emboj.7600293

Kaibara C, Matsui T, Hisabori T, Yoshida M (1996) Structural asymmetry of F1-ATPase caused by the $\gamma$ subunit generates a high affinity nucleotide binding site. J Biol Chem 271:2433-2438

Keis S, Stocker A, Dimroth P, Cook GM (2006) Inhibition of ATP hydrolysis by thermoalkaliphilic F1Fo-ATP synthase is controlled by the $\mathrm{C}$ terminus of the $\varepsilon$ subunit. J Bacteriol 188:3796-3804. doi:10. 1128/JB.00040-06

Kodera N, Ando T (2014) The path to visualization of walking myosin V by high-speed atomic force microscopy. Biophys Rev 6:237-260. doi:10.1007/s12551-014-0141-7

Komoriya Y, Ariga T, Iino R, Imamura H, Okuno D, Noji H (2012) Principal role of the arginine finger in rotary catalysis of F1-ATPase. J Biol Chem 287:15134-15142. doi:10.1074/jbc.M111.328153

Konno H, Murakami-Fuse T, Fujii F, Koyama F, Ueoka-Nakanishi H, Pack CG, Kinjo M, Hisabori T (2006) The regulator of the F1 motor: inhibition of rotation of cyanobacterial F1-ATPase by the $\varepsilon$ subunit. EMBO J 25:4596-4604

Kühlbrandt W, Davies KM (2016) Rotary ATPases: a new twist to an ancient machine. Trends Biochem Sci 41:106-116. doi:10.1016/j. tibs.2015.10.006 
Lane N, Martin WF (2012) The origin of membrane bioenergetics. Cell 151:1406-1416. doi:10.1016/j.cell.2012.11.050

Le Trong I, Freitag S, Klumb LA, Chu V, Stayton PS, Stenkamp RE (2003) Structural studies of hydrogen bonds in the high-affinity streptavidin-biotin complex: mutations of amino acids interacting with the ureido oxygen of biotin. Acta Crystallogr D Biol Crystallogr 59:1567-1573

Li CB, Ueno H, Watanabe R, Noji H, Komatsuzaki T (2015) ATP hydrolysis assists phosphate release and promotes reaction ordering in F1ATPase. Nat Commun 6:10223. doi:10.1038/ncomms 10223

Masaike T, Koyama-Horibe F, Oiwa K, Yoshida M, Nishizaka T (2008) Cooperative three-step motions in catalytic subunits of F(1)-ATPase correlate with $80^{\circ}$ and $40^{\circ}$ substep rotations. Nat Struct Mol Biol 15: 1326-1333. doi:10.1038/nsmb.1510

Matthies D, Preiss L, Klyszejko AL, Muller DJ, Cook GM, Vonck J, Meier T (2009) The c13 ring from a thermoalkaliphilic ATP synthase reveals an extended diameter due to a special structural region. J Mol Biol 388:611-618. doi:10.1016/j.jmb.2009.03.052

McLachlin DT, Bestard JA, Dunn SD (1998) The $b$ and delta subunits of the Escherichia coli ATP synthase interact via residues in their Cterminal regions. J Biol Chem 273:15162-15168

McMillan DGG, Keis S, Dimroth P, Cook GM (2007) A specific adaptation in the $a$ subunit of thermoalkaliphilic F1FO-ATP synthase enables ATP synthesis at high $\mathrm{pH}$ but not at neutral $\mathrm{pH}$ values. J Biol Chem 282:17395-17404. doi:10.1074/jbc.M611709200

McMillan DGG, Keis S, Berney M, Cook GM (2009) Nonfermentative thermoalkaliphilic growth is restricted to alkaline environments. Appl Environ Microbiol 75:7649-7654. doi:10.1128/AEM.01639-09

McMillan DGG, Watanabe R, Ueno H, Cook GM, Noji H (2016) Biophysical characterization of a thermoalkaliphilic molecular motor with a high stepping torque gives insight into evolutionary ATP synthase adaptation. J Biol Chem 291:23965-23977. doi:10.1074/ jbc.M116.743633

Meier T, Polzer P, Diederichs K, Welte W, Dimroth P (2005a) Structure of the rotor ring of F-type Na+-ATPase from Ilyobacter tartaricus. Science (New York, NY) 308:659-662. doi:10.1126/science. 1111199

Meier T, Yu J, Raschle T, Henzen F, Dimroth P, Muller DJ (2005b) Structural evidence for a constant c11 ring stoichiometry in the sodium F-ATP synthase. FEBS J 272:5474-5483. doi:10.1111/j.17424658.2005.04940.x

Menz RI, Walker JE, Leslie AG (2001) Structure of bovine mitochondrial F(1)-ATPase with nucleotide bound to all three catalytic sites: implications for the mechanism of rotary catalysis. Cell 106:331-341

Morales-Rios E, Montgomery MG, Leslie AG, Walker JE (2015) Structure of ATP synthase from Paracoccus denitrificans determined by X-ray crystallography at 4.0 Å resolution. Proc Natl Acad Sci U S A 112:13231-13236. doi:10.1073/pnas. 1517542112

Mukherjee S, Warshel A (2015) Dissecting the role of the $\gamma$-subunit in the rotary-chemical coupling and torque generation of F1-ATPase. Proc Natl Acad Sci U S A 112:2746-2751. doi:10.1073/pnas. 1500979112

Müller M, Gumbiowski K, Cherepanov DA, Winkler S, Junge W, Engelbrecht S, Pänke O (2004) Rotary F1-ATPase. Is the Cterminus of subunit $\gamma$ fixed or mobile? Eur J Biochem 271:39143922. doi:10.1111/j.1432-1033.2004.04328.x

Nishizaka T, Oiwa K, Noji H, Kimura S, Muneyuki E, Yoshida M, Kinosita K Jr (2004) Chemomechanical coupling in F1-ATPase revealed by simultaneous observation of nucleotide kinetics and rotation. Nat Struct Mol Biol 11:142-148. doi:10.1038/nsmb721

Noji H, Yoshida M (2001) The rotary machine in the cell, ATP synthase. J Biol Chem 276:1665-1668. doi:10.1074/jbc.R000021200

Noji H, Yasuda R, Yoshida M, Kinosita K Jr (1997) Direct observation of the rotation of F1-ATPase. Nature 386:299-302. doi:10.1038/ $386299 \mathrm{a} 0$
Noji H, Bald D, Yasuda R, Itoh H, Yoshida M, Kinosita K Jr (2001) Purine but not pyrimidine nucleotides support rotation of $\mathrm{F}(1)$ ATPase. J Biol Chem 276:25480-25486. doi:10.1074/jbc. M102200200

Ogilvie I, Aggeler R, Capaldi RA (1997) Cross-linking of the $\delta$ subunit to one of the three $\alpha$ subunits has no effect on functioning, as expected if $\delta$ is a part of the stator that links the F1 and F0 parts of the Escherichia coli ATP synthase. J Biol Chem 272:16652-16656

Okazaki KI, Hummer G (2015) Elasticity, friction, and pathway of $\gamma$ subunit rotation in FoF1-ATP synthase. Proc Natl Acad Sci U S A 112:10720-10725. doi:10.1073/pnas.1500691112

Okuno D, Iino R, Noji H (2011) Rotation and structure of FoF1-ATP synthase. J Biochem 149:655-664. doi:10.1093/jb/mvr049

Omote H, Sambonmatsu N, Saito K, Sambongi Y, Iwamoto-Kihara A, Yanagida T, Wada Y, Futai M (1999) The $\gamma$-subunit rotation and torque generation in F1-ATPase from wild-type or uncoupled mutant Escherichia coli. Proc Natl Acad Sci U S A 96:7780-7784

Oster G, Wang H (2000) Reverse engineering a protein: the mechanochemistry of ATP synthase. Biochim Biophys Acta 1458:482-510

Oster G, Wang H, Grabe M (2000) How Fo-ATPase generates rotary torque. Philos Trans R Soc Lond Ser B Biol Sci 355:523-528. doi: 10.1098/rstb.2000.0593

Pänke O, Cherepanov DA, Gumbiowski K, Engelbrecht S, Junge W (2001) Viscoelastic dynamics of actin filaments coupled to rotary F-ATPase: angular torque profile of the enzyme. Biophys $\mathrm{J}$ 81: 1220-1233. doi:10.1016/S0006-3495(01)75780-3

Petersen J, Förster K, Turina P, Gräber P (2012) Comparison of the H+/ ATP ratios of the H+ATP synthases from yeast and from chloroplast. Proc Natl Acad Sci U S A 109:11150-11155. doi:10.1073/ pnas. 1202799109

Pogoryelov D, Yu J, Meier T, Vonck J, Dimroth P, Müller DJ (2005) The c15 ring of the Spirulina platensis F-ATP synthase: F1/F0 symmetry mismatch is not obligatory. EMBO Rep 6:1040-1044. doi:10.1038/ sj.embor.7400517

Pogoryelov D, Reichen C, Klyszejko AL, Brunisholz R, Muller DJ, Dimroth P, Meier T (2007) The oligomeric state of $c$ rings from cyanobacterial F-ATP synthases varies from 13 to 15 . J Bacteriol 189:5895-5902. doi:10.1128/JB.00581-07

Pogoryelov D, Yildiz Ö, Faraldo-Gómez JD, Meier T (2009) Highresolution structure of the rotor ring of a proton-dependent ATP synthase. Nat Struct Mol Biol 16:1068-1073. doi:10.1038/nsmb. 1678

Pogoryelov D, Klyszejko AL, Krasnoselska GO, Heller EM, Leone V, Langer JD, Vonck J, Müller DJ, Faraldo-Gómez JD, Meier T (2012) Engineering rotor ring stoichiometries in the ATP synthase. Proc Natl Acad Sci U S A 109:E1599-E1608. doi:10.1073/pnas. 1120027109

Rees DM, Montgomery MG, Leslie AG, Walker JE (2012) Structural evidence of a new catalytic intermediate in the pathway of ATP hydrolysis by F1-ATPase from bovine heart mitochondria. Proc Natl Acad Sci U S A 109:11139-11143. doi:10.1073/pnas. 1207587109

Rodgers AJ, Capaldi RA (1998) The second stalk composed of the b- and $\delta$-subunits connects F0 to F1 via an $\alpha$-subunit in the Escherichia coli ATP synthase. J Biol Chem 273:29406-29410

Rondelez Y, Tresset G, Nakashima T, Kato-Yamada Y, Fujita H, Takeuchi S, Noji H (2005) Highly coupled ATP synthesis by F1-ATPase single molecules. Nature 433:773-777. doi:10.1038/nature03277

Saita EI, Suzuki T, Kinosita K Jr, Yoshida M (2015) Simple mechanism whereby the F1-ATPase motor rotates with near-perfect chemomechanical energy conversion. Proc Natl Acad Sci U S A 112:9626-9631. doi:10.1073/pnas. 1422885112

Schulenberg B, Aggeler R, Murray J, Capaldi RA (1999) The $\gamma \mathcal{\varepsilon}$-c subunit interface in the ATP synthase of Escherichia coli. Cross-linking of the $\varepsilon$ subunit to the $\mathrm{c}$ subunit ring does not impair enzyme 
function, that of $\gamma$ to $\mathrm{c}$ subunits leads to uncoupling. J Biol Chem 274:34233-34237

Senior AE, Al-Shawi MK (1992) Further examination of seventeen mutations in Escherichia coli F1-ATPase beta-subunit. J Biol Chem 267:21471-21478

Shimabukuro K, Yasuda R, Muneyuki E, Hara KY, Kinosita K Jr, Yoshida M (2003) Catalysis and rotation of F1 motor: cleavage of ATP at the catalytic site occurs in $1 \mathrm{~ms}$ before $40^{\circ}$ substep rotation. Proc Natl Acad Sci U S A 100:14731-14736. doi:10.1073/pnas. 2434983100

Shimo-Kon R, Muneyuki E, Sakai H, Adachi K, Yoshida M, Kinosita K Jr (2010) Chemo-mechanical coupling in F(1)-ATPase revealed by catalytic site occupancy during catalysis. Biophys J 98:1227-1236. doi:10.1016/j.bpj.2009.11.050

Sielaff H, Martin J, Singh D, Biuković G, Grüber G, Frasch WD (2016) Power stroke angular velocity profiles of archaeal A-ATP synthase versus thermophilic and mesophilic F-ATP synthase molecular motors. J Biol Chem 291:25351-25363. doi:10.1074/jbc.M116. 745240

Sobti M, Smits C, Wong AS, Ishmukhametov R, Stock D, Sandin S, Stewart AG (2016) Cryo-EM structures of the autoinhibited E. coli ATP synthase in three rotational states. eLife 5:e21598. doi:10.7554/ eLife. 21598

Spetzler D, York J, Daniel D, Fromme R, Lowry D, Frasch W (2006) Microsecond time scale rotation measurements of single F1-ATPase molecules. Biochemistry 45:3117-3124. doi:10.1021/bi052363n

Stock D, Leslie AG, Walker JE (1999) Molecular architecture of the rotary motor in ATP synthase. Science (New York, NY) 286: $1700-1705$

Stocker A, Keis S, Cook GM, Dimroth P (2005) Purification, crystallization, and properties of F1-ATPase complexes from the thermoalkaliphilic Bacillus sp. strain TA2.A1. J Struct Biol 152: 140-145

Suzuki T, Tanaka K, Wakabayashi C, Saita EI, Yoshida M (2014) Chemomechanical coupling of human mitochondrial F1-ATPase motor. Nat Chem Biol 10:930-936. doi:10.1038/nchembio.1635

Svoboda K, Block SM (1994) Force and velocity measured for single kinesin molecules. Cell 77:773-784

Symersky J, Pagadala V, Osowski D, Krah A, Meier T, Faraldo-Gómez JD, Mueller DM (2012) Structure of the c(10) ring of the yeast mitochondrial ATP synthase in the open conformation. Nat Struct Mol Biol 19:485-491. doi:10.1038/nsmb.2284

Tanigawara M, Tabata KV, Ito Y, Ito J, Watanabe R, Ueno H, Ikeguchi M, Noji H (2012) Role of the DELSEED loop in torque transmission of F1-ATPase. Biophys J 103:970-978. doi:10.1016/j.bpj.2012.06.054

Tsunoda SP, Rodgers AJ, Aggeler R, Wilce MC, Yoshida M, Capaldi RA (2001) Large conformational changes of the $\varepsilon$ subunit in the bacterial F1F0 ATP synthase provide a ratchet action to regulate this rotary motor enzyme. Proc Natl Acad Sci U S A 98:6560-6564. doi:10.1073/pnas.111128098

Turina P, Petersen J, Gräber P (2016) Thermodynamics of proton transport coupled ATP synthesis. Biochim Biophys Acta 1857:653-664. doi:10.1016/j.bbabio.2016.02.019

Uchihashi T, Iino R, Ando T, Noji H (2011) High-speed atomic force microscopy reveals rotary catalysis of rotorless F-ATPase. Science (New York, NY) 333:755-758. doi:10.1126/science.1205510

Ueno H, Nishikawa S, Iino R, Tabata KV, Sakakihara S, Yanagida T, Noji H (2010) Simple dark-field microscopy with nanometer spatial precision and microsecond temporal resolution. Biophys J 98:2014 2023. doi:10.1016/j.bpj.2010.01.011

Ueno H, Minagawa Y, Hara M, Rahman S, Yamato I, Muneyuki E, Noji H, Murata T, Iino R (2014) Torque generation of Enterococcus hirae V-ATPase. J Biol Chem 289:31212-31223. doi:10.1074/jbc.M114. 598177
Usukura E, Suzuki T, Furuike S, Soga N, Saita EI, Hisabori T, Kinosita K, Yoshida M (2012) Torque generation and utilization in motor enzyme F0F1-ATP synthase: half-torque F1 with short-sized pushrod helix and reduced ATP synthesis by half-torque F0F1. J Biol Chem 287:1884-1891. doi:10.1074/jbc.M111.305938

Vahidi S, Bi Y, Dunn SD, Konermann L (2016) Load-dependent destabilization of the $\gamma$-rotor shaft in FOF1 ATP synthase revealed by hydrogen/deuterium-exchange mass spectrometry. Proc Natl Acad Sci U S A 113:2412-2417. doi:10.1073/pnas.1520464113

van Raaij MJ, Abrahams JP, Leslie AG, Walker JE (1996) The structure of bovine F1-ATPase complexed with the antibiotic inhibitor aurovertin B. Proc Natl Acad Sci U S A 93:6913-6917

Vollmar M, Schlieper D, Winn M, Büchner C, Groth G (2009) Structure of the $\mathrm{c} 14$ rotor ring of the proton translocating chloroplast ATP synthase. J Biol Chem 284:18228-18235. doi:10.1074/jbc.M109. 006916

Wächter A, Bi Y, Dunn SD, Cain BD, Sielaff H, Wintermann F, Engelbrecht S, Junge W (2011) Two rotary motors in F-ATP synthase are elastically coupled by a flexible rotor and a stiff stator stalk. Proc Natl Acad Sci U S A 108:3924-3929. doi:10.1073/pnas. 1011581108

Walker JE, Saraste M, Runswick MJ, Gay NJ (1982) Distantly related sequences in the alpha- and beta-subunits of ATP synthase, myosin, kinases and other ATP-requiring enzymes and a common nucleotide binding fold. EMBO J 1:945-951

Watanabe R, Iino R, Noji H (2010) Phosphate release in F1-ATPase catalytic cycle follows ADP release. Nat Chem Biol 6:814-820. doi:10.1038/nchembio.443

Watanabe R, Okuno D, Sakakihara S, Shimabukuro K, Iino R, Yoshida M, Noji H (2012) Mechanical modulation of catalytic power on F1ATPase. Nat Chem Biol 8:86-92. doi:10.1038/nchembio.715

Watanabe R, Tabata KV, Iino R, Ueno H, Iwamoto M, Oiki S, Noji H (2013) Biased Brownian stepping rotation of FoF1-ATP synthase driven by proton motive force. Nat Commun 4:1631

Watanabe R, Matsukage Y, Yukawa A, Tabata KV, Noji H (2014) Robustness of the rotary catalysis mechanism of F1-ATPase. J Biol Chem 289:19331-19340. doi:10.1074/jbc.M114.569905

Watt IN, Montgomery MG, Runswick MJ, Leslie AG, Walker JE (2010) Bioenergetic cost of making an adenosine triphosphate molecule in animal mitochondria. Proc Natl Acad Sci U S A 107:16823-16827. doi:10.1073/pnas.1011099107

Watts SD, Zhang Y, Fillingame RH, Capaldi RA (1995) The $\gamma$ subunit in the Escherichia coli ATP synthase complex (ECF1F0) extends through the stalk and contacts the $\mathrm{c}$ subunits of the F0 part. FEBS Lett 368:235-238

Weber J, Senior AE (1997) Catalytic mechanism of F1-ATPase. Biochim Biophys Acta 1319:19-58

Yagi H, Kajiwara N, Iwabuchi T, Izumi K, Yoshida M, Akutsu H (2009) Stepwise propagation of the ATP-induced conformational change of the F1-ATPase $\beta$ subunit revealed by NMR. J Biol Chem 284:2374 2382. doi:10.1074/jbc.M808212200

Yasuda R, Noji H, Kinosita K Jr, Yoshida M (1998) F1-ATPase is a highly efficient molecular motor that rotates with discrete $120^{\circ}$ steps. Cell 93:1117-1124

Yasuda R, Noji H, Yoshida M, Kinosita K Jr, Itoh H (2001) Resolution of distinct rotational substeps by submillisecond kinetic analysis of F1ATPase. Nature 410:898-904. doi:10.1038/35073513

Yildiz A, Forkey JN, McKinney SA, Ha T, Goldman YE, Selvin PR (2003) Myosin V walks hand-over-hand: single fluorophore imaging with 1.5-nm localization. Science (New York, NY) 300:20612065. doi:10.1126/science. 1084398

Yoshida M, Muneyuki E, Hisabori T (2001) ATP synthase - a marvellous rotary engine of the cell. Nat Rev Mol Cell Biol 2:669-677. doi:10. $1038 / 35089509$ 